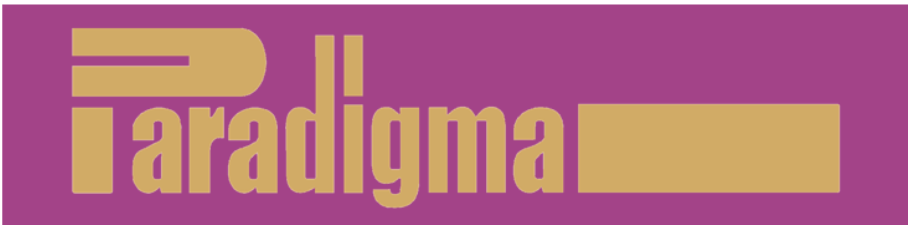

Revista del Centro de Investigaciones Educacionales Paradigma Depósito Legal AR2019000054 - ISSN N 2665 - 0126

Volumen XLI, Edición Cuadragésimo Aniversario; Junio de 2020

\title{
EL ROSTRO HUMANO DE LA EDICIÓN POR EL CUADRAGÉSIMO ANIVERSARIO DE LA REVISTA PARADIGMA (1980-2020)
}

\section{THE HUMAN FACE OF THE EDITION FOR THE FORTIETH ANNIVERSARY OF THE REVISTA PARADIGMA MAGAZINE (1980-2020)}

Cuatro décadas, cuarenta años, cuarenta y un artículos, ochenta y tres autores y autoras de cuarenta y tres instituciones y doce países.

Muchas gracias, en nombre de todos los que hacen posible esta publicación, por acompañarnos en este momento tan especial.

\section{Dr. Fredy González \\ Director - Editor}

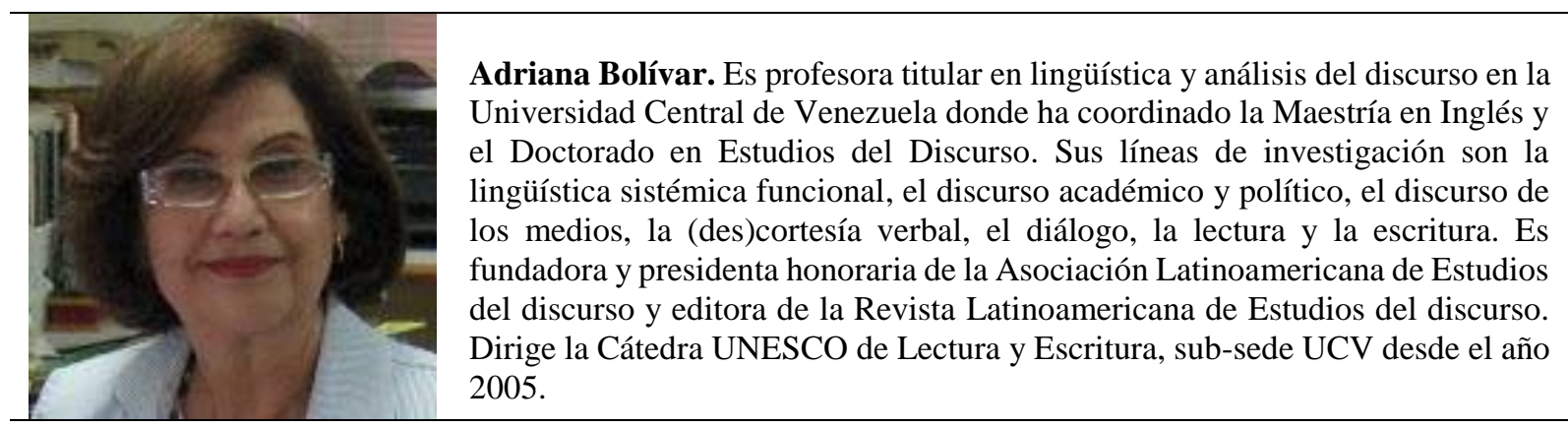

Adriana Breda. Doutora em Educação em Ciências e Matemática pela Pontifícia Universidade Católica do Rio Grande do Sul (PUCRS). Possui Mestrado em Educação em Ciências e Matemática pela Pontifícia Universidade Católica do Rio Grande do Sul (PUCRS). Atualmente é professora e pesquisadora na Universitat de Barcelona (UB, Espanha). É membro do Comité Latinoamericano de Educación Matemática (CLAME).

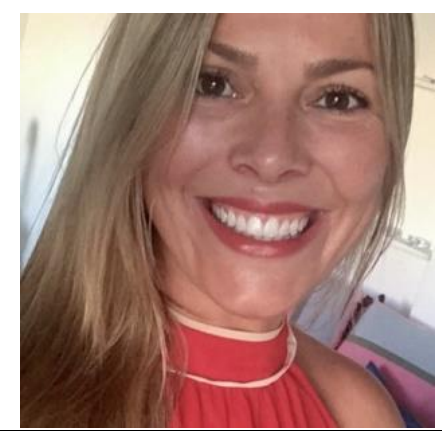




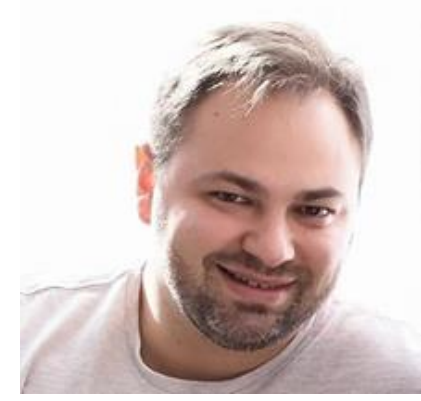

Alexandre Ausani Huff. possui Pós-graduação em Psicopedagogia Clínica e Institucional (2013), Supervisão e Orientação escolar (2015), ambos pela Universidade La Salle - UNILASALLE/Canoas e em Educação Infantil (2019) pela Universidade Positivo; Mestrado em Ensino de Ciências e Matemática (2018) pela Universidade Luterana do Brasil - ULBRA/RS. Atualmente é professor de Matemática do ensino fundamental na Prefeitura Municipal de São Leopoldo e professor de Matemática do ensino fundamental na Prefeitura Municipal de Porto Alegre. Doutorando em Ensino de Ciências e Matemática pela Universidade Luterana do Brasil - ULBRA/RS.

Alicia Fernández-Oliveras. Licenciada en Física, Diplomada en Óptica y Doctora en Física, por la Universidad de Granada. Profesora Ayudante Doctora del Departamento de Didáctica de las Ciencias Experimentales de la Facultad de Ciencias de la Educación de la Universidad de Granada.

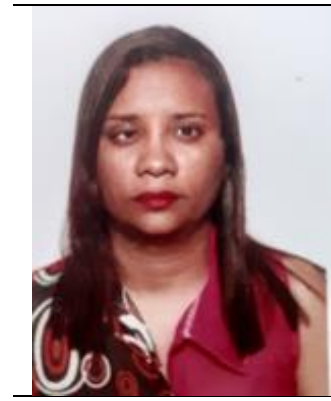

Ana Cristina Bolívar Orellana. Doctora en Pedagogía del Discurso por la Universidad Pedagógica Experimental Libertador (UPEL, Venezuela). Actualmente encargada de la División de Investigación. y Coordinadora del “Grupo Interdisciplinario de Investigación Educativa Recinto Urania Montás” del ISFODOSU (Recinto Urania Montás)-República Dominicana. Docente e investigadora (Línea: Diálogo de saberes interdisciplinarios) de la UPEL (IPREM, Venezuela) y del Centro de Investigaciones Educacionales Paradigma (UPEL Maracay)

Ana Lúcia Manrique. Possui graduação em Matemática pela Universidade de São Paulo (1987), mestrado em Ensino de Matemática (1994) e doutorado em Educação (Psicologia da Educação) (2003), ambos pela Pontifícia Universidade Católica de São Paulo e Pos-Doutorado no Programa de Pós-graduação em Educação da PUC/RJ (Pós-Doc Júnior CNPq) (2008). É professora da Pontifícia Universidade Católica de São Paulo..

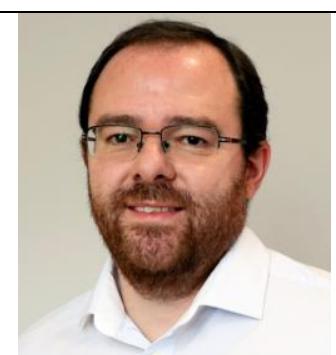

Antonio Francisco Roldán López de Hierro, es profesor titular en el Departamento de Estadística e Investigación Operativa de la Universidad de Granada (España). Es doctor en Ciencias Matemáticas por la Universidad de Granada y doctor en Estadística por la Universidad de Jaén España). Sus líneas de investigación principales son la regresión con datos imprecisos, los números difusos en computación y la toma de decisiones en ambientes de incertidumbre, además de la Didáctica de la Estadística y de la Matemática.

Arno Bayer. possui doutorado em Educação pela Universidad Pontificia de Salamanca Espanha (1997). Atualmente é professor titular da Universidade Luterana do Brasil e professor orientador do Programa de Pós-Graduação em Ensino de Ciências e Matemática (PPGECIM) - Mestrado e Doutorado. Avaliador de Curso de Graduação e Avaliador Institucional do Sistema Nacional de Avaliação da Educação Superior.Tem experiência na área de Matemática, com ênfase em Matemática, atuando principalmente nos seguintes temas: Educação Estatística, Ensino Médio, Estatística, Educação Matemática e formação de professores.

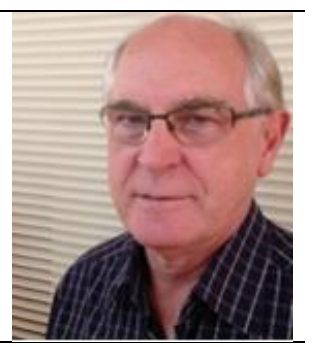




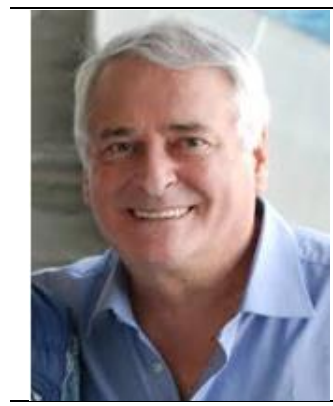

Bruno D'Amore se graduó en Matemáticas, Filosofía y Pedagogía de la Universidad de Bolonia perfeccionado en matemáticas elementales desde un punto de vista superior. Doctorado en Educación Matemática; en 2013 la Universidad de Chipre. Galardonado con un doctorado honorario en Ciencias Sociales y Educación. Profesor titular de Didáctica de las Matemáticas en el Departamento de Matemáticas de la Universidad de Bolonia. Ha colaborado activamente con el Ministerio de Educación pública italiana (MPI). Actualmente es profesor experto titular en el Doctorado Interinstitucional en Educación (DIE), (énfasis matemática), en la Universidad Distrital Francisco José de Caldas, Bogotá, Colombia.

Carlos Aldemir Farias da Silva é professor da Universidade Federal do Pará, onde atua no Programa de Pós-graduação em Educação em Ciências e Matemáticas. Doutor em Ciências Sociais (Antropologia) pela Pontifícia Universidade Católica de São Paulo. Membro efetivo da ABA e da ANPED. Editor de revistas científicas.

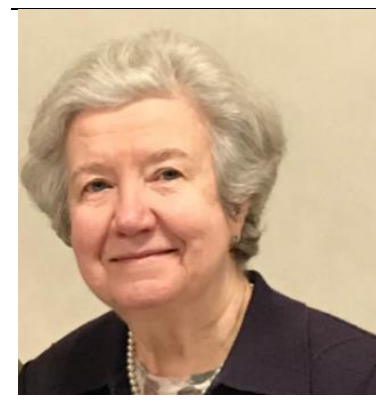

Carmen Batanero. Fue catedrática en el Departamento de Didáctica de la Matemática de la Universidad de Granada, España y tras su jubilación es profesora colaboradora en dicho departamento. Sus publicaciones incluyen trabajos de investigación y libros dirigidos a maestros. Ella ayudó a establecer un grupo estocástico en PME (Psicología de la Educación Matemática), CERME y algunas conferencias latinoamericanas. Fue Presidenta de IASE durante el período 20012003, miembro del Comité Ejecutivo (1997-2007) y miembro del Comité Ejecutivo de ICMI (2003-2006). Es miembro vitalicio del IASE. Actualmente es editora asociada en la revista Statistics Education Reseach Journal y miembro del panel editorial de otras revistas. https://www.ugr.es/ batanero/

Carmen Teresa Kaiber. Doutorado em Ciências da Educação pela Universidade Pontifícia de Salamanca, Espanha. Graduação em Matemática pela Universidade do Vale do Rio dos Sinos, São Leopoldo/RS, Brasil. Professora Titular do Programa de

Pós-Graduação em Ensino de Ciências e Matemática - PPGECIM e do Curso de Matemática da Universidade Luterana do Brasil - ULBRA. Pesquisadora do Grupo de Estudos Curriculares em Educação Matemática (GECEM). https://orcid.org/0000-0003-1883-230X. E-mail: kaiber@ulbra.br

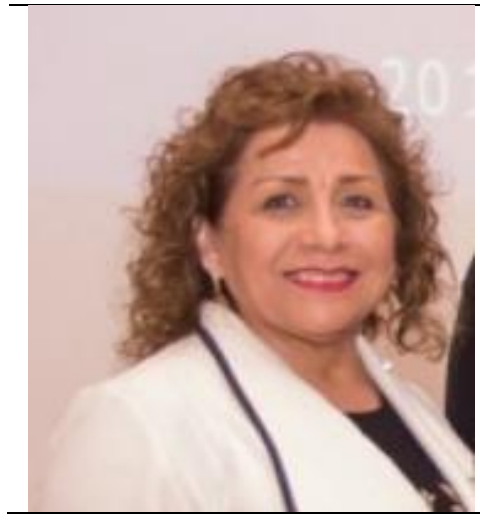

Cecilia Susana Sanhueza Cartes, Bachelor en Educación de la Universidad Austral de Chile, Educadora de Párvulo de la Universidad de Chile. Supervisora de la Junta Nacional de Jardines Infantiles en la totalidad de la Región del Bio-Bio. Directora de la carrera de Educación Parvularia en la Universidad de Los Lagos del 1983-1993. Académica jornada completa en la Universidad Católica de Temuco desde 1994 a la fecha. Ocupó el cargo de Secretaria Académica de la Escuela de Educación Parvularia. Con participación en el Centro de Capacitación de la Universidad Católica de Temuco en proyectos FOSIS. Expositora en congresos de especialidad con publicaciones ligadas a la didáctica de la educación parvularia y las prácticas pedagógicas. 

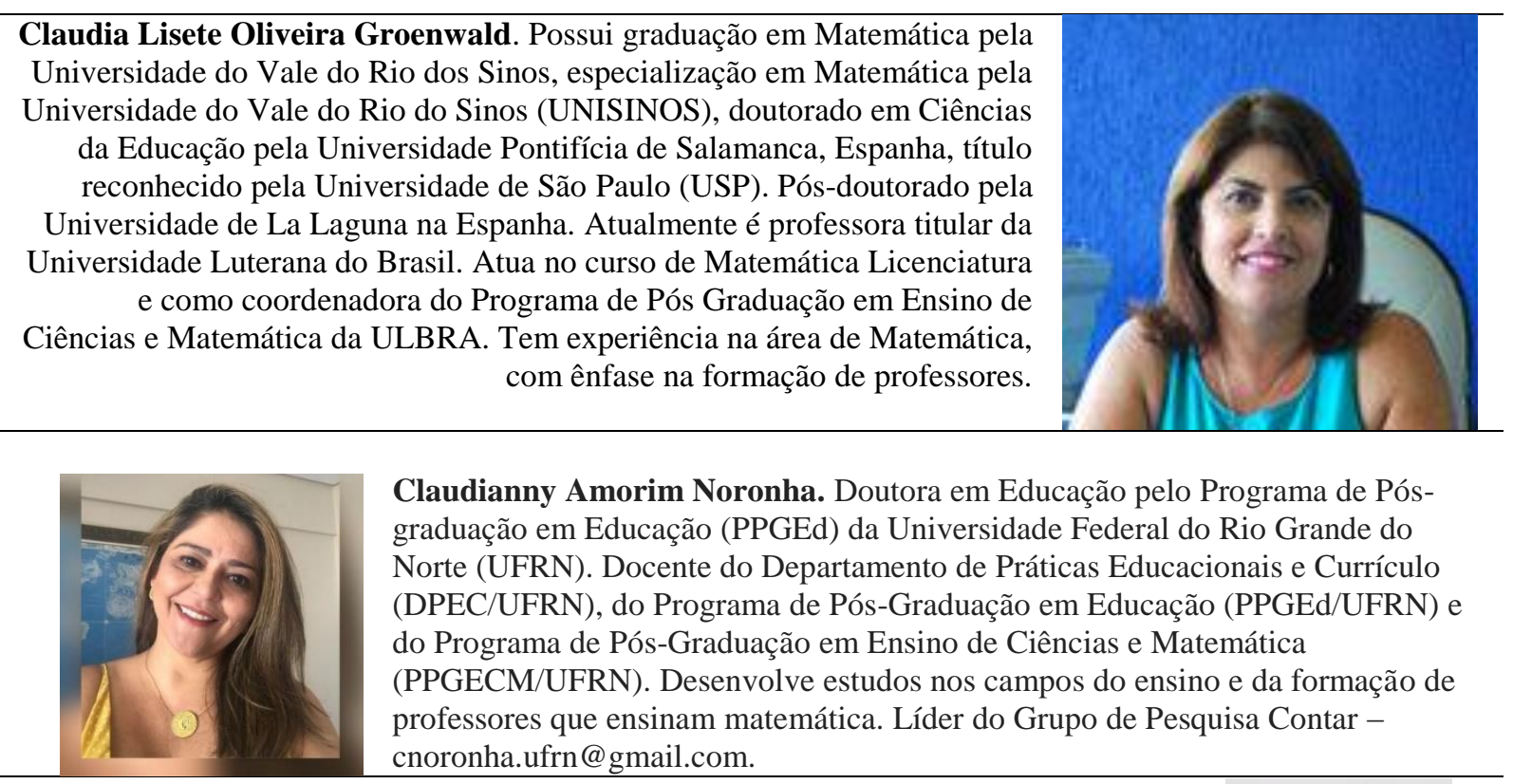

Claudianny Amorim Noronha. Doutora em Educação pelo Programa de Pósgraduação em Educação (PPGEd) da Universidade Federal do Rio Grande do Norte (UFRN). Docente do Departamento de Práticas Educacionais e Currículo (DPEC/UFRN), do Programa de Pós-Graduação em Educação (PPGEd/UFRN) e do Programa de Pós-Graduação em Ensino de Ciências e Matemática (PPGECM/UFRN). Desenvolve estudos nos campos do ensino e da formação de professores que ensinam matemática. Líder do Grupo de Pesquisa Contar cnoronha.ufrn@gmail.com.

Cleissiane Aguido Gotardo. Mestranda na Universidade Estadual do Paraná (UNESPAR), Campus Paranavaí- Mestrado em Ensino: Formação Docente Interdisciplinar (PPIFOR); Graduada em Pedagogia pela Universidade Estadual do Paraná (UNESPAR), Campus Paranavaí, e Pesquisadora do Grupo de Estudos e Pesquisas Trabalho e Educação na Sociabilidade do Capital (GEPTESC/UNESPAR/CNPQ).

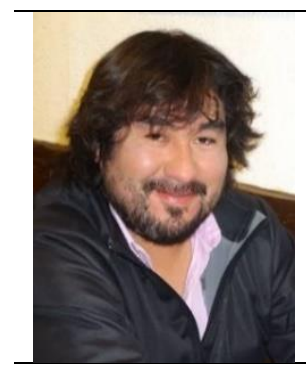

Danilo Díaz-Levicoy. Profesor de Matemática y Computación (ULAGOS). Máster en Didáctica de la Matemática (UGR). Doctor en Ciencias de la Educación (UGR). Académico de la Facultad de Ciencias Básicas, Universidad Católica del Maule. Línea de Investigación: Didáctica de la Matemática y la Estadística. Orcid: https://orcid.org/00000001-8371-7899 ResearchGate: https://www.researchgate.net/profile/Danilo_DiazLevicoy dddiaz01@ @otmail.com

David Antonio da Costa. Doutor em Educação Matemática pela PUC/SP. Atualmente é professor associado do Departamento de Metodologia de Ensino e professor credenciado no Programa de Pós-Graduação em Educação Científica e Tecnológica da Universidade

Federal de Santa Catarina. É pesquisador lider do Grupo de Pesquisa de História da Educação Matemática - GHEMAT-SC. Membro fundador da GHEMAT-BRASIL: Grupo Associado de Estudos e Pesquisas sobre História da Educação Matemática: 1o. secretário (Mandato 2018-2021)

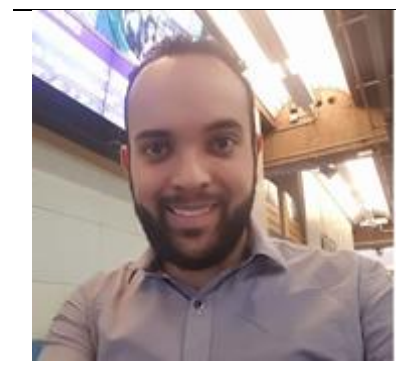

Douglas da Silva Tinti. Doutor em Educação Matemática pela Pontifícia Universidade Católica de São Paulo (PUC/SP), com período sanduíche na Universidade de Sevilha/Espanha. É professor do Departamento de Educação Matemática (DEEMA) da Universidade Federal de Ouro Preto. Tem experiência nas áreas de Educação e Ensino, com ênfase em Educação Matemática, atuando principalmente nos seguintes temas: Formação de professores que ensinam matemática, iniciação à docência, aprendizagem da docência, Comunidades de Prática, Práticas de Ensino e Estágio Curricular Supervisionado. 
Elsa Midori Shimazaki. Doutora. Professora do Programa de Pós-Graduação em Educação. Universidade Estadual de Maringá, PR, Brasil. Membro dos Grupos de Pesquisa: O Ensino e a Inclusão de Pessoas com Deficiência e Interação e Linguagem. Email:emshimazaki@uem.br. https://orcid.org/0000-0002-2225-5667. Endereço postal: Rua Floriano Peixoto, 1340, apto 102. Maringá-PR

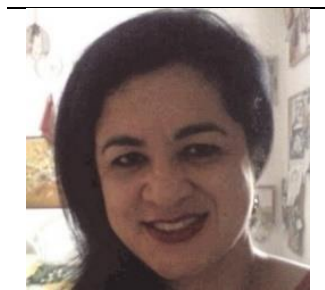

Evelyn Cristina Arreaza Páez. Doctorante en Educación. Profesora Asociado de la Facultad de Ciencias de la Educación de la Universidad de Carabobo, Investigadora PEII Nivel B. Formadora de Tutores Virtuales. Profesora de postgrado en TIC. Investigadora sobre las Tecnologías de la Información y Comunicación y los Procesos de Enseñanza de la Lengua y la Literatura. En la actualidad, profesora de español en Estados Unidos de América.

Everton Bedin. Pós-doutor e Doutor em Educação em Ciências (UFRGS). Mestre em Educação Química (UFU). Especialista em Gestão Educacional (UFSM) e em Tecnologias da Informação e Comunicação (FURG). Graduado em Licenciatura em Química (UPF). Professor no Departamento de Química e no Programa de Mestrado Profissional em Química em Rede Nacional (PROFQUI) da Universidade Federal do Paraná (UFPR). https://orcid.org/0000-0002-5636-0908 http://lattes.cnpq.br/9498564582615440
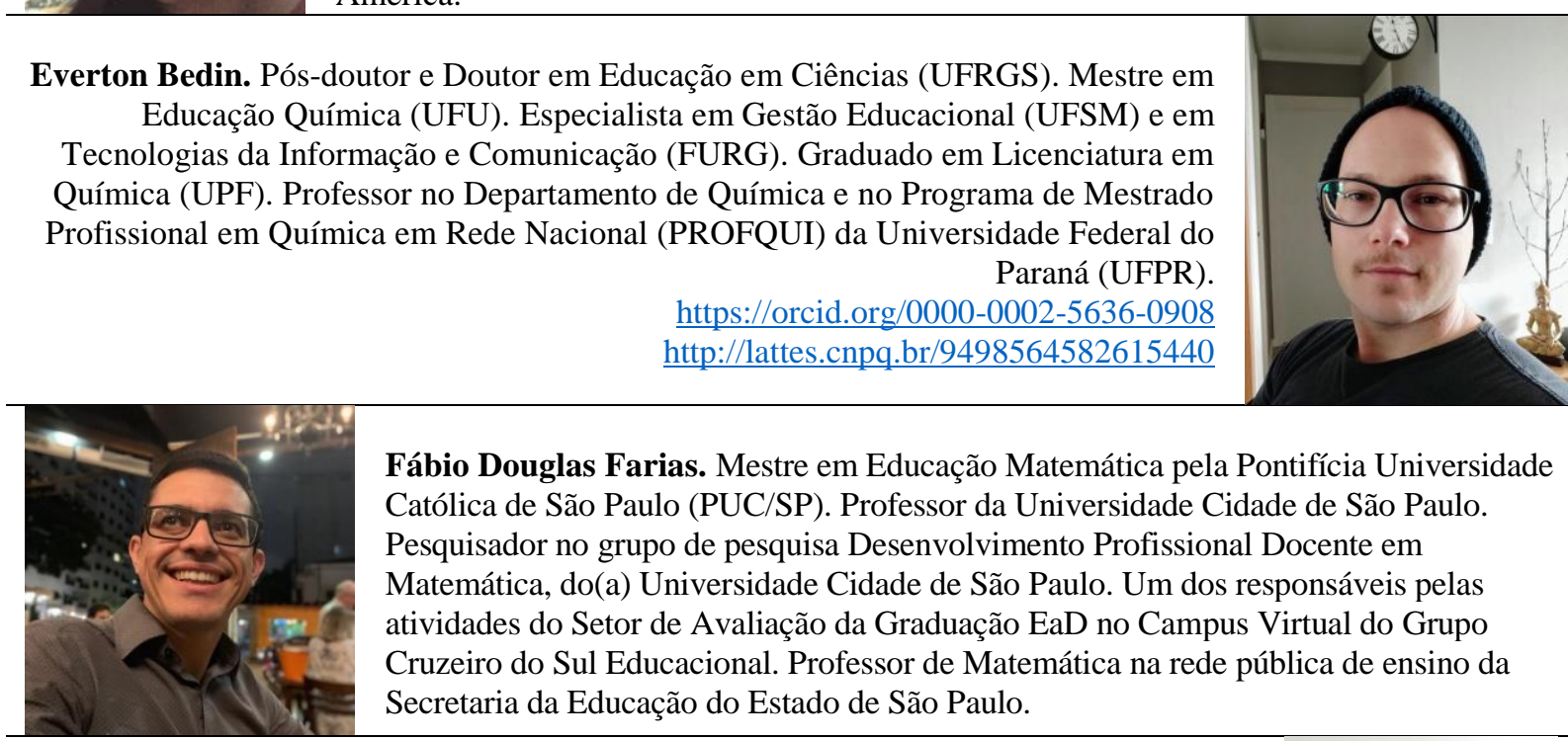

Fábio Douglas Farias. Mestre em Educação Matemática pela Pontifícia Universidade Católica de São Paulo (PUC/SP). Professor da Universidade Cidade de São Paulo. Pesquisador no grupo de pesquisa Desenvolvimento Profissional Docente em Matemática, do(a) Universidade Cidade de São Paulo. Um dos responsáveis pelas atividades do Setor de Avaliação da Graduação EaD no Campus Virtual do Grupo Cruzeiro do Sul Educacional. Professor de Matemática na rede pública de ensino da Secretaria da Educação do Estado de São Paulo.

Fernando Luís de Rosso. possui Mestrado em Ensino de Ciências e Matemática pela Universidade Luterana do Brasil - ULBRA (2018). Atualmente é Doutorando em Ensino de Ciências e Matemática pela Universidade Luterana do Brasil - ULBRA e Professor de Matemática na rede pública estadual do Rio Grande do Sul. Tem experiência na formação de trabalhadores pelo programa Jovem Aprendiz e Cursos Técnicos nas áreas de Matemática, Matemática Financeira e Estatística.
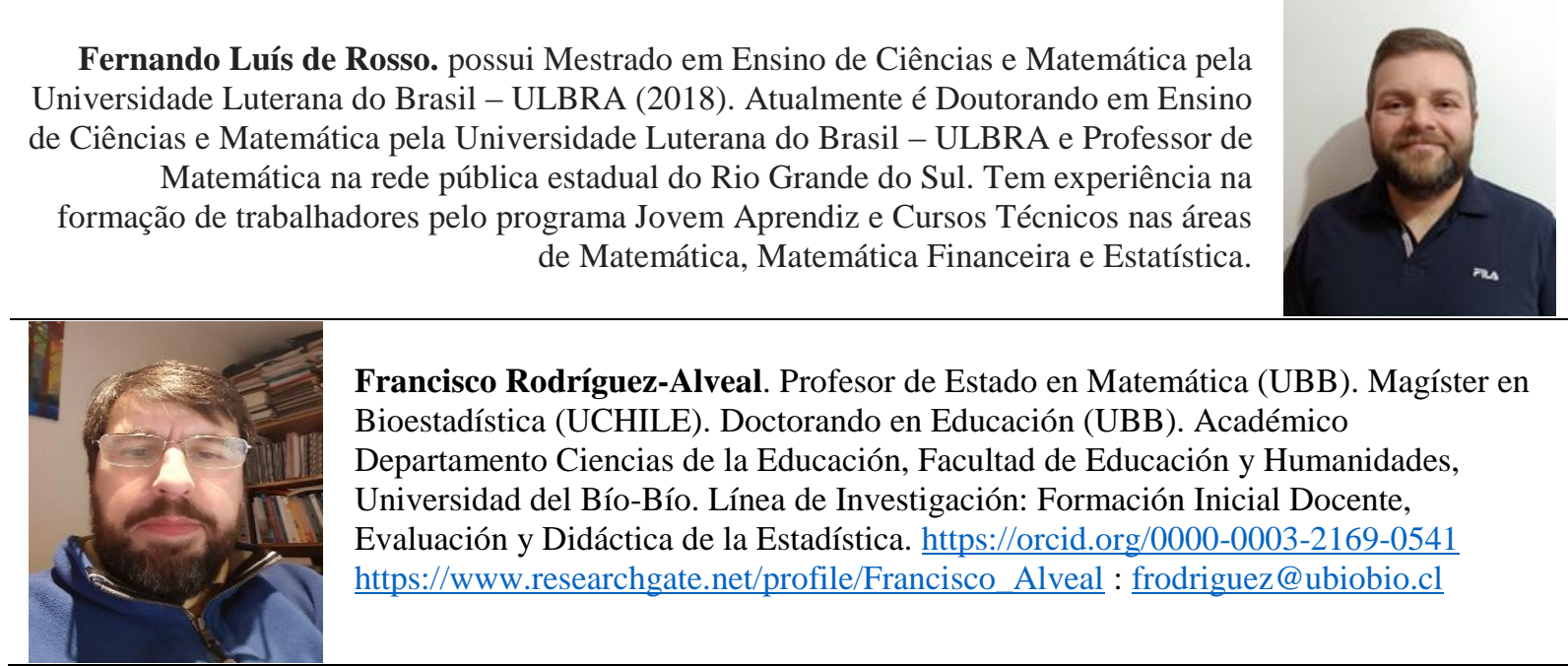

Francisco Rodríguez-Alveal. Profesor de Estado en Matemática (UBB). Magíster en Bioestadística (UCHILE). Doctorando en Educación (UBB). Académico Departamento Ciencias de la Educación, Facultad de Educación y Humanidades, Universidad del Bío-Bío. Línea de Investigación: Formación Inicial Docente, Evaluación y Didáctica de la Estadística. https://orcid.org/0000-0003-2169-0541 https://www.researchgate.net/profile/Francisco_Alveal : frodriguez@ubiobio.cl 
Freddy F. Rojas Velásquez. Doctor en educación. Master in Curriculum and Instruction. Estudios en educación Superior Universitaria y Psicología. Profesor de Biología y Química. Profesor Titular Jubilado de la Universidad Simón Bolívar. Profesor de Postgrado en Metodología de la Investigación, Teorías de Aprendizajes, Educación y Tecnología. Miembro del programa de estímulo a la Innovación e Investigación (PEII). Área de Investigación: Educación y Familia, estilos de Aprendizaje y Estilos de Pensamiento, Educación y Tecnología. frojas@usb.ve
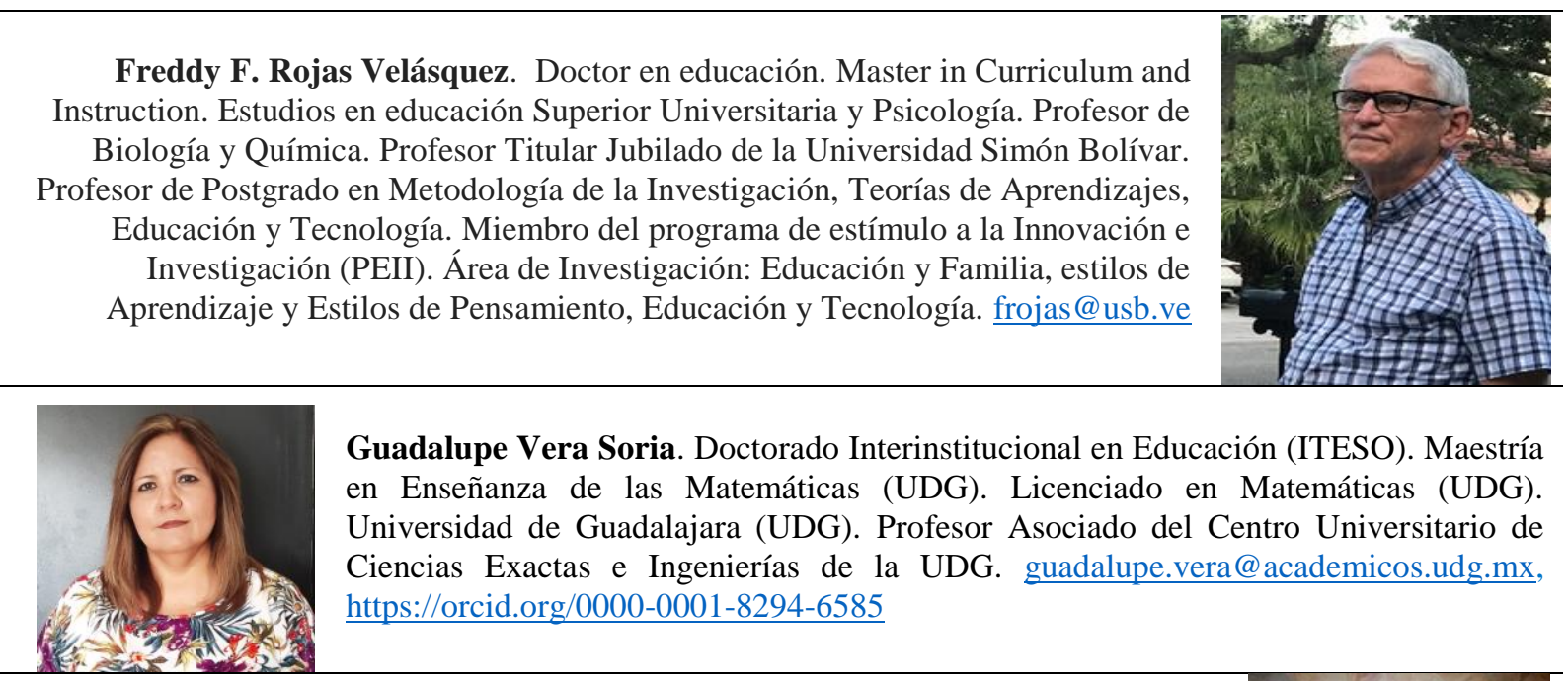

Guadalupe Vera Soria. Doctorado Interinstitucional en Educación (ITESO). Maestría en Enseñanza de las Matemáticas (UDG). Licenciado en Matemáticas (UDG). Universidad de Guadalajara (UDG). Profesor Asociado del Centro Universitario de Ciencias Exactas e Ingenierías de la UDG. guadalupe.vera@academicos.udg.mx, https://orcid.org/0000-0001-8294-6585

Haydee Guillermina Páez. Doctor en Educación. Profesora Titular jubilada y miembro del Centro de Investigaciones Educativas de la Facultad de Ciencias de la Educación de la Universidad de Carabobo. Actual Rectora de la Universidad José Antonio Páez, San Diego, estado Carabobo, Venezuela.

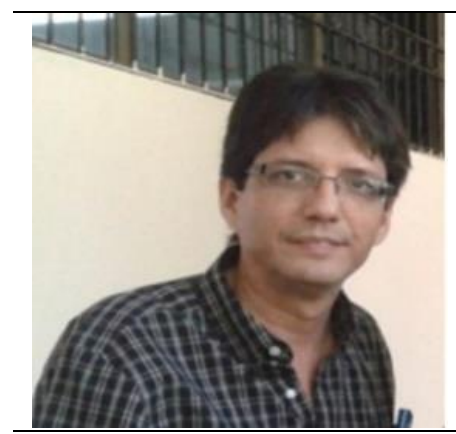

Hugo Parra Sandoval. Profesor Titular y Emérito de la Universidad del Zulia. Área de investigación Conocimiento profesional del profesor de Matemáticas y Matemática en Contexto. Asesor de programas de formación de docentes en matemática y profesor invitado de la Universidad Pedagógica Experimental Libertador- Barquisimeto, Venezuela y de la Universidad del Atlántico en Barranquilla, Colombia. Ex presidente de la Asociación Venezolana de Educación Matemática - ASOVEMAT (2013-2016), Presidente de la Federación Iberoamericana de Educación Matemática-FISEM (2014-2016). Vocal por Sudamérica (2008-2012) y Secretario (2016-2020) del Comité Latinoamericano de Matemática Educativa - CLAME

Iran Abreu Mendes. Professor Titular da Universidade Federal do Pará (UFPA) onde atua no Programam de Pós-graduação em Educação em Ciências e Matemáticas.

É Pesquisador 1C do CNPq. Licenciado em Matemática com Mestrado e Doutorado em Educação e Pós-Doutorado em Educação Matemática. Com experiência no ensino de Cálculo, Geometria Euclidiana e Analítica, História da Matemática, Ensino de Matemática e Etnomatemática.

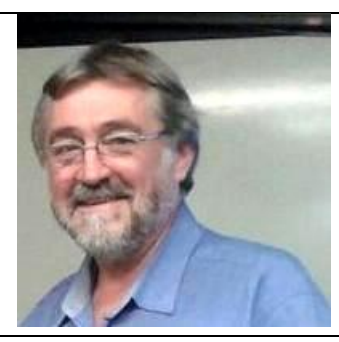

José Claudio Del Pino. Pós-doutor em Ensino de Química (Universidade de Aveiro). Doutor em Biomassa e Mestre em Bioquímica (UFRGS). Graduado em Química (UFRGS). Professor da Universidade do Vale do Taquari (UNIVATES). Professor no Programa de Pós-Graduação em Ensino da Universidade do Vale do Taquari (UNIVATES). Linha de Investigação: Ensino de química e formação de professores. https://orcid.org/0000-0002-8321-9774 http://lattes.cnpq.br/2152799270731771 delpinojc@yahoo.com.br 
José Ortiz Buitrago. Universidad de Carabobo, Campus La Morita, Venezuela. Profesor de Matemática, con Doctorado en Didáctica de la Matemática, por la Universidad de Granada, España. Coordinador de la Línea de Investigación Educación Matemática: Pensamiento Numérico y Algebraico, adscrita a la Unidad de Investigación del Ciclo Básico de la Facultad de Ciencias Económicas y Sociales. Integrante del Núcleo de Investigación en Educación Matemática. ortizbuitrago@gmail.com
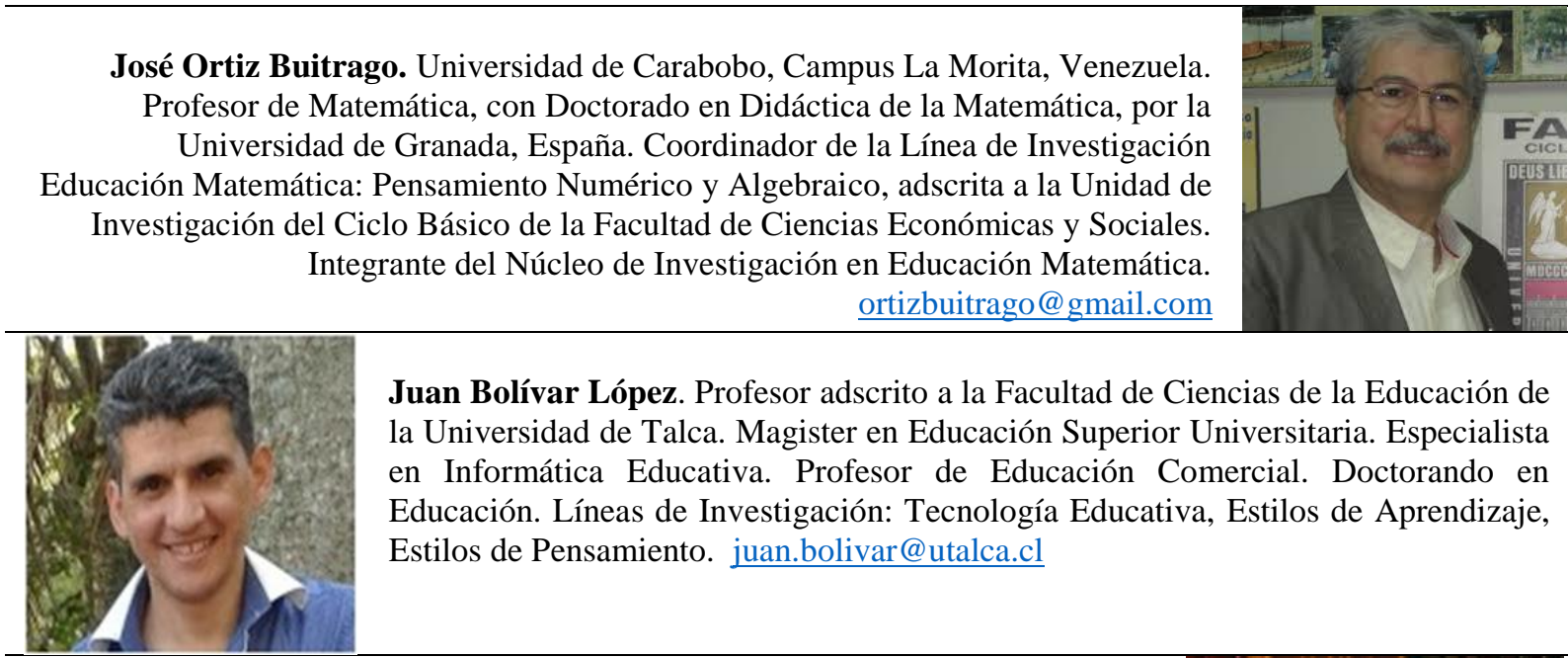

Juan Bolívar López. Profesor adscrito a la Facultad de Ciencias de la Educación de la Universidad de Talca. Magister en Educación Superior Universitaria. Especialista en Informática Educativa. Profesor de Educación Comercial. Doctorando en Educación. Líneas de Investigación: Tecnología Educativa, Estilos de Aprendizaje, Estilos de Pensamiento.juan.bolivar@utalca.cl

Juan D. Godino actualmente trabaja en el Departamento de Didáctica de la Matemática, Universidad de Granada. Investiga en educación matemática. Su proyecto actual es desarrollar y aplicar el Enfoque Onto-Semiótico al conocimiento matemático y la instrucción (OSA) (Enfoque Ontosemiótico del Conocimiento y la Instrucción Matemática (EOS). Ver sitio web, http://enfoqueontosemiotico.ugr.es
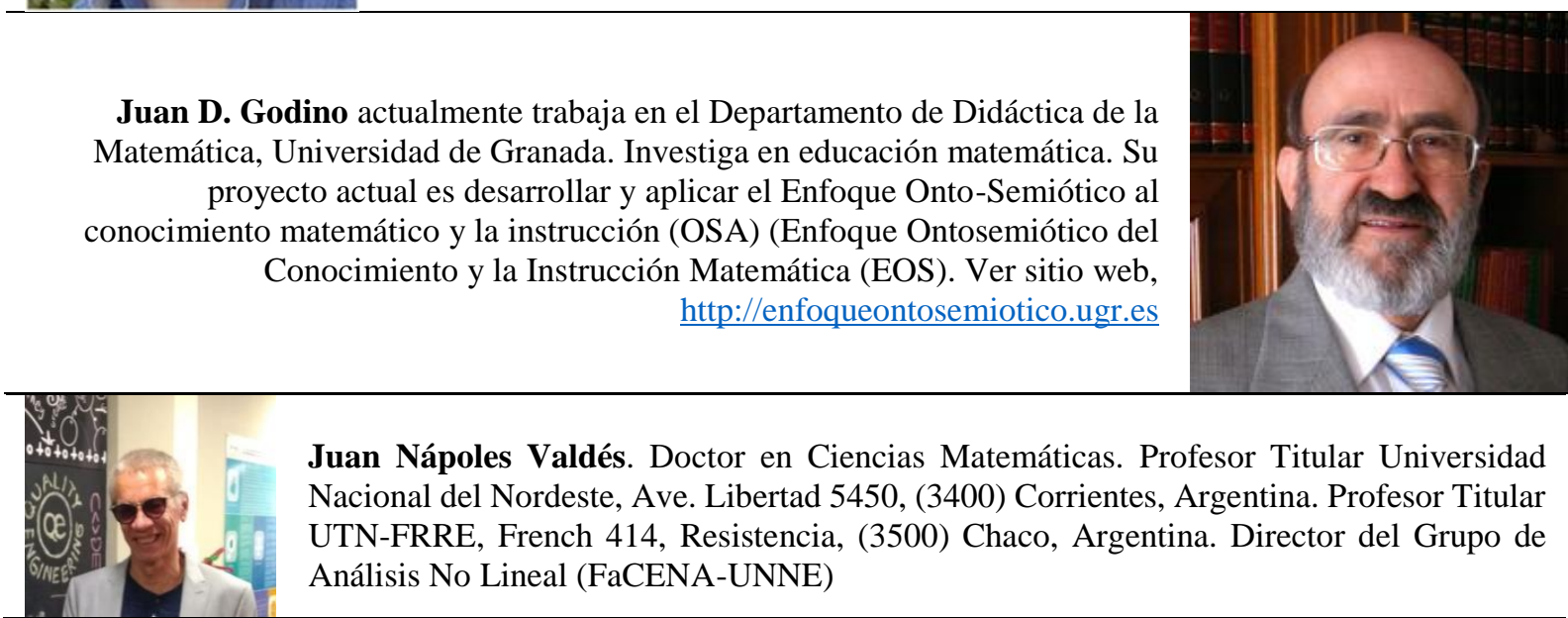

Juan Nápoles Valdés. Doctor en Ciencias Matemáticas. Profesor Titular Universidad Nacional del Nordeste, Ave. Libertad 5450, (3400) Corrientes, Argentina. Profesor Titular UTN-FRRE, French 414, Resistencia, (3500) Chaco, Argentina. Director del Grupo de Análisis No Lineal (FaCENA-UNNE)

Julio R. González Bello. Profesor Titular (Jubilado-Activo). Universidad de Carabobo. Facultad de Ciencias de la Educación. Departamento de Orientación. Doctor en Educación Universidad de Carabobo). Master en Orientación (Oklahoma State University). Licenciado en Educación. Mención Orientación. Fundador de la Red Latinoamericana de Profesionales de la Orientación. ExVicepresidente y Miembro de la Junta Directiva de la Internacional Association for Educational and Vocational Guidance.

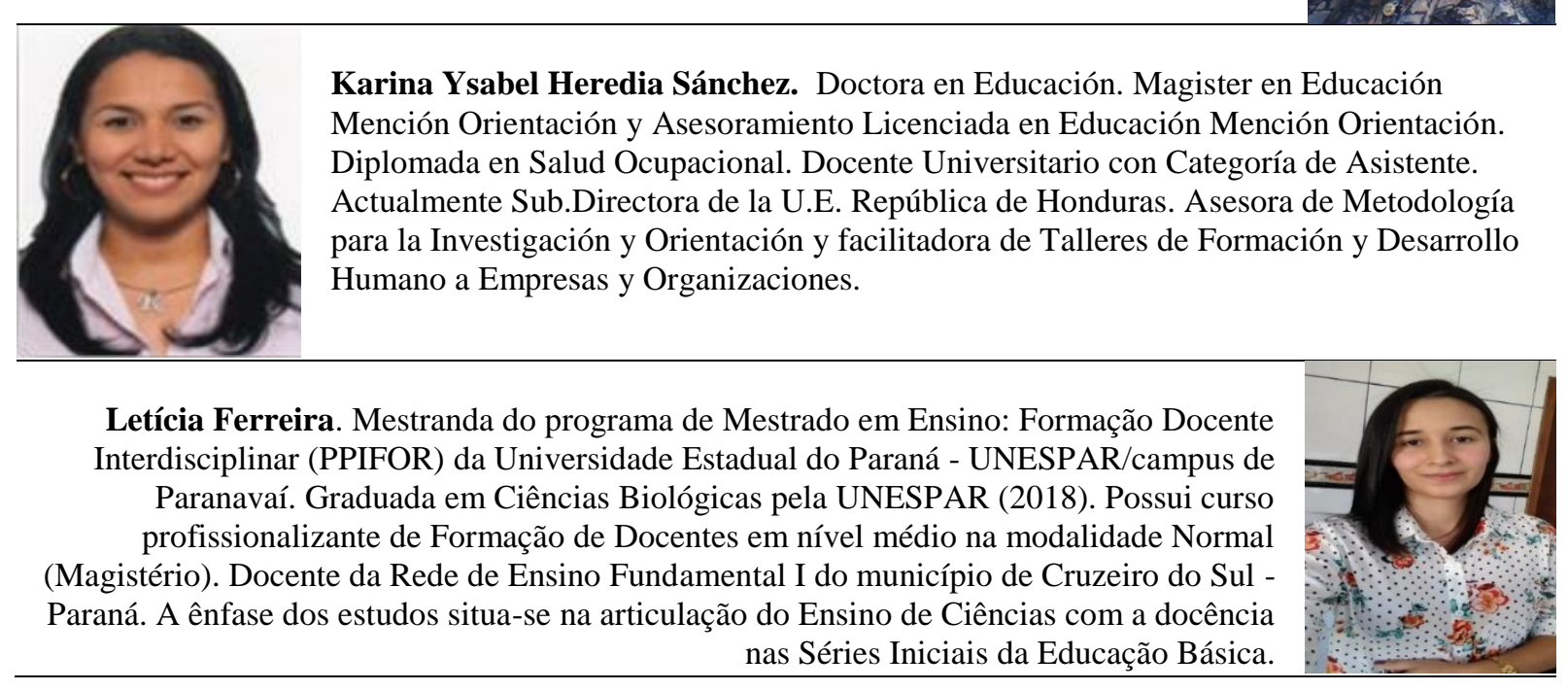




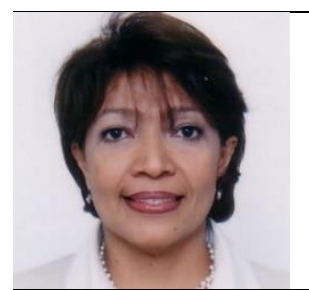

Ligia Sánchez. Doctora en Sociología. Docente investigadora en la Universidad de Carabobo, Campus La Morita (UC). Directora de la Unidad de Investigación del Ciclo Básico, Facultad de Ciencias Económicas y Sociales (FACES). Universidad de Carabobo (Venezuela).ligia.uc@gmail.com. https://orcid.org/0000-0003-1410-9309

Lizzet Morales-Garcia. Licenciado en Matemáticas (UAGro). Maestría en Ciencias: Área Matemática Educativa (UAGro). Doctoranda en Ciencias con Especialidad en Matemática Educativa (UAGro). Línea de Investigación: Didáctica y Epistemología de la Matemática. https://orcid.org/0000-0002-2295-2278 https://www.researchgate.net/profile/Lizzet_MoralesGarcia lmgarcia@uagro.mx

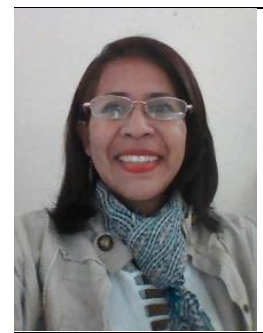

Lourdes Ángela Díaz Blanca. Doctora en Lingüística por la Universidad de los Andes (ULA-Venezuela). Docente categoría Titular a Dedicación Exclusiva. UPEL, Instituto Pedagógico de Mejoramiento Profesional de Magisterio IMPM. Núcleo Mérida. Adscrita al Centro de Investigaciones Lingǘsticas y Literarias Hugo Obregón Muñoz (IPMAR) y al Núcleo de Investigación Extensión Mérida. Correo electrónico: ludiblan40@ gmail.com. ORCID https://orcid.org/0000-0003-2709-883X. Mérida, Venezuela.

Luanna Priscila da Silva Gomes. Doutora em Educação pelo Programa de Pós Graduação em Educação (PPGEd/UFRN). Mestre em Educação (PPGEd/UFRN). Participa do CONTAR (http://www.grupocontar.com.br/) - Grupo de Pesquisa em ensino da matemática e da língua portuguesa/UFRN/CNPQ (http://dgp.cnpq.br/dgp/espelhogrupo/2573628422946154). Professora efetiva do Núcleo de Educação da Infância - Colégio de Aplicação - NEI/CAp - UFRN . Estuda a linguagem matemática na infância. https://orcid.org/0000-0002-7157-6870

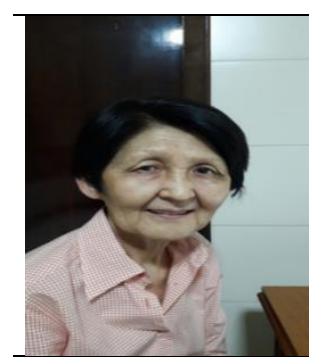

Lucila Akiko Nagashima. Docente do programa de Mestrado em Ensino: Formação Docente Interdisciplinar da Universidade Estadual do Paraná - UNESPAR/campus de Paranavaí. Graduação em Ciências pela UNESPAR e em Química pela Universidade Estadual de Maringá/UEM. Mestrado (2005) e Doutorado (2010) pela Universidade Estadual de Maringá. O destaque dos estudos situa-se na esfera da Educação Superior em articulação com a Educação Básica, especificamente no Ensino de Ciências.

Luis R. Pino-Fan (http://www.lrpino-fan.com/), doctor en Didáctica de la Matemática por la Universidad de Granada, España. Actualmente es Académico del Departamento de Ciencias Exactas de la Universidad de Los Lagos, Chile, Director del Programa de Magíster en Educación Matemática. Es Director de la Sociedad Chilena de Educación Matemática (SOCHIEM). En 2015, propuso junto con el Dr. Juan Godino, el Modelo del Conocimiento Didáctico-Matemático (CDM), el cual sigue siendo muy usado a nivel internacional para caracterizar y desarrollar conocimientos y competencias clave para la práctica profesional del profesor de matemáticas.

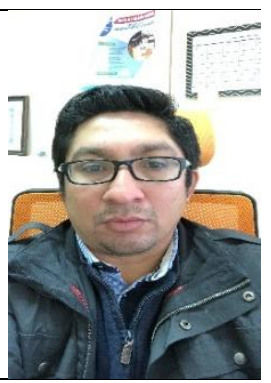




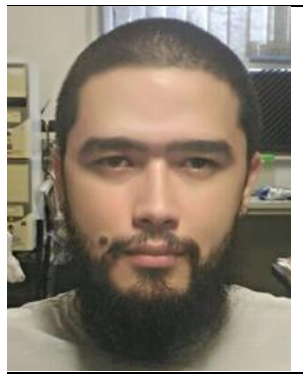

Luiz Ricardo Nakamura. Graduado em estatística (UNESP), mestre e doutor em ciências, com área de concentração em estatística e experimentação agronômica (USP). Atualmente é professor adjunto do Departamento de Informática e Estatística da Universidade Federal de Santa Catarina. Possui experiência na área de Probabilidade e Estatística, com ênfase em Probabilidade e Estatística Aplicadas, atuando principalmente nos seguintes temas: análise de dados, modelos semiparamétricos, modelagem estatística, aprendizagem estatística e análise multivariada.

Mabel Rodríguez. Licenciada y Doctora de la Universidad de Buenos Aires, área Matemática. Se desempeña en el Instituto del Desarrollo Humano de la Universidad Nacional de General Sarmiento como investigadora-docente donde dirige la Especialización en Didáctica de las Ciencias y el Profesorado Universitario de Educación Superior en Matemática. Área de investigación: Educación Matemática y Formación de Profesores.

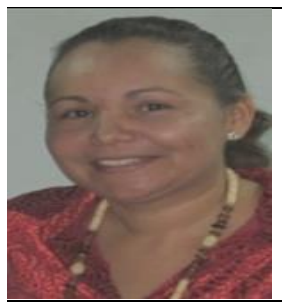

Maidelis Herrera. Licda. en Educación. Magister en Investigación Educativa. Miembro activo de la Unidad de Investigación del Ciclo Básico, Facultad de Ciencias Económicas y Sociales (FACES-UC). Universidad de Carabobo (Venezuela) maidelis16@gmail.com. https://orcid.org/0000-0003-0634-3779

Mairene Sánchez. Profesora en Lingüística y Literatura. Especialista en Gerencia Educativa. Docente de Investigación básica y Coordinadora Cátedra de Proyecto I y II de Investigación, Universidad Bicentenaria de Aragua. (Venezuela) mairenesanchez@gmail.com https://orcid.org/0000-0001-9795-3075

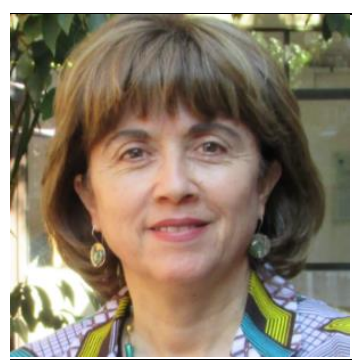

Marcela Parraguez González. Doctorado en Matemática Educativa (CICATAMéxico). Magíster en Matemáticas (PUCV). Licenciado en Matemáticas (PUCV). Profesor de Matemáticas (PUCV). Pontificia Universidad Católica de Valparaíso (PUCV). Directora del Instituto de Matemáticas de la PUCV. Docente jerarquizada del Instituto de Matemáticas de la PUCV. Integrante del Consejo Directivo del Comité Latinoamericano de Matemática Educativa, CLAME, en calidad de Vocal por Sudamérica. marcela.parraguez@ pucv.cl, https://orcid.org/0000-0002-6164-3056

Marcelo Franco Leão. Posui Doutorado em Educação em Ciências (UFRGS). Mestre em Ensino (UNIVATES). Especialização em Orientação Educacional (Dom Alberto) e em Relações Raciais na Educação e na Sociedade Brasileira (UFMT). Graduação em Licenciatura em Química (UNISC) e em Física (UNEMAT). Professor do Departamento de Ensino do IFMT Campus Confresa. Membro do Grupo de Pesquisa Ensino de Ciências e Matemática no Baixo Araguaia (EnCiMa). 


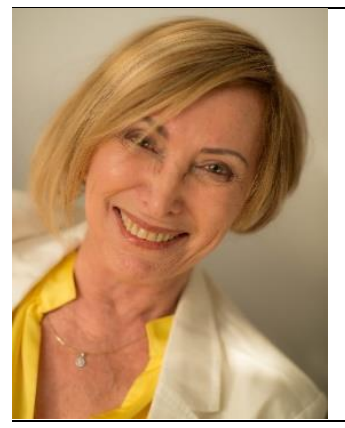

Maria Aparecida Viggiani Bicudo. Professora Titular da Universidade Estadual Paulista Júlio de Mesquita Filho (Unesp) em Filosofia da Educação (Atualmente aposentada). É professora e pesquisadora do Programa de Pós-Graduação em Educação Matemática da UNESP, Câmpus de Rio Claro. Co-Editora do Boletim de Educação Matemática - BOLEMA. Editora da Revista Pesquisa Qualitativa www.sepq.org.br/revista . Bolsista do CNPq - 1-A. Presidente da Sociedade de Estudos e Pesquisa Qualitativos - www.sepq.org.br. Autora de livros e de artigos científico-acadêmicos. Site: www.mariabicudo.com.br

María Burgos. Profesora Contratada Doctora en la Universidad de Granada. Doctora en Matemáticas por la Universidad de Almería y Doctora en Didáctica de la Matemática por la Universidad de Granada. Mis líneas de investigación fundamentales son la teoría de la educación matemática, formación de profesores, y el razonamiento proporcional y algebraico.
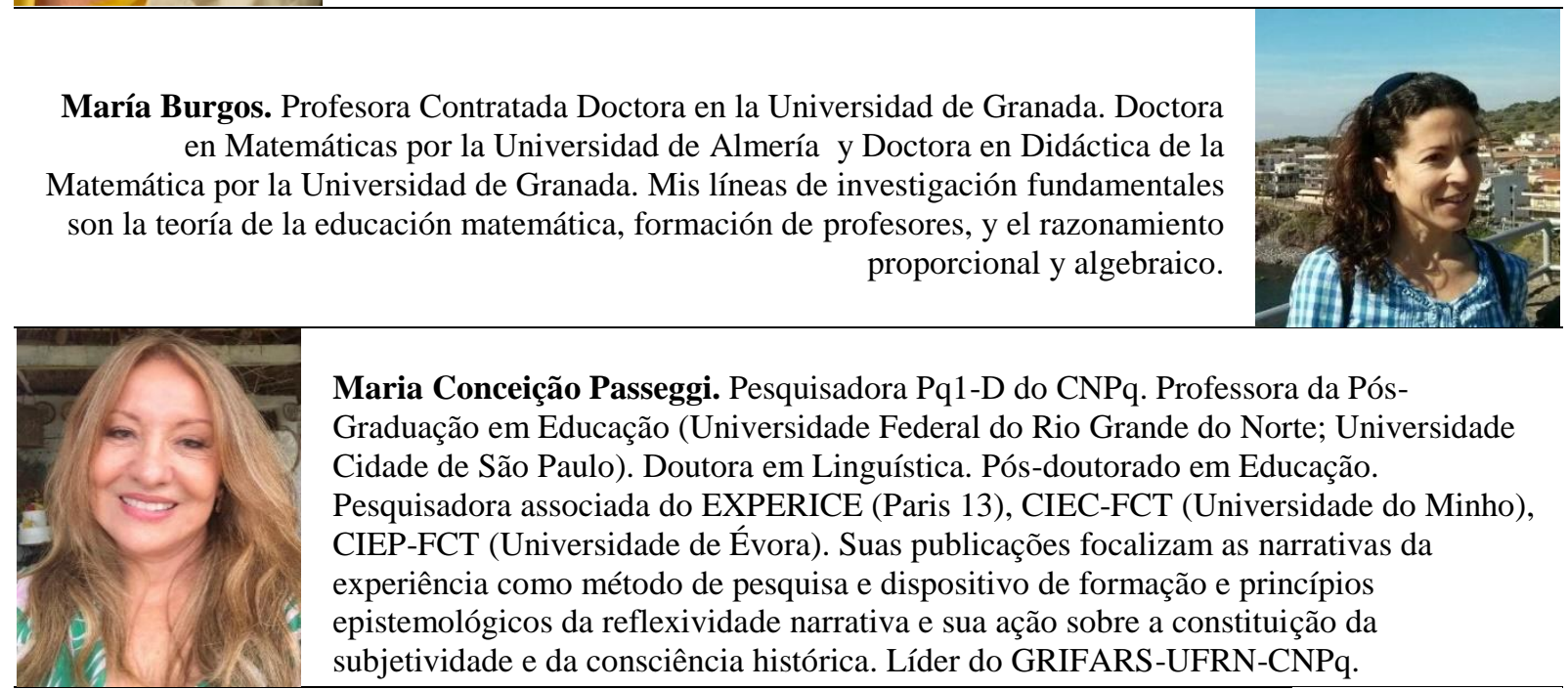

Maria Conceição Passeggi. Pesquisadora Pq1-D do CNPq. Professora da PósGraduação em Educação (Universidade Federal do Rio Grande do Norte; Universidade Cidade de São Paulo). Doutora em Linguística. Pós-doutorado em Educação. Pesquisadora associada do EXPERICE (Paris 13), CIEC-FCT (Universidade do Minho), CIEP-FCT (Universidade de Évora). Suas publicações focalizam as narrativas da experiência como método de pesquisa e dispositivo de formação e princípios epistemológicos da reflexividade narrativa e sua ação sobre a constituição da subjetividade e da consciência histórica. Líder do GRIFARS-UFRN-ÇPq.

María José Espigares-Gámez. Graduada en Educación Infantil, Máster en Didáctica de la Matemática, por la Universidad de Granada y Estudiante de Doctorado del Programa de Ciencias de la Educación en la Universidad de Granada.

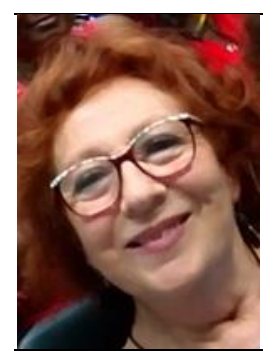

María Luisa Oliveras. Licenciada en Matemáticas y Doctora en Educación Matemática por la Universidad de Granada. Catedrática del Departamento de Didáctica de las Matemáticas de la Facultad de Ciencias de la Educación de la Universidad de Granada. Ex Presidenta del ISGEM International Study Group on Ethnomathematics.

María Susana Harringhton Martínez. Doctora en Educación por la Universidad Pedagógica Experimental Libertador (UPEL, Venezuela). Docente categoría Asociado a Dedicación Exclusiva. UPEL, Instituto Pedagógico Rural El Mácaro, "Luis Fermín".

Coordinadora de la Línea: Investigar desde la educación: Diálogo de saberes interdisciplinarios. Correo electrónico susanhm23@gmail.com, ORCID https://orcid.org/0000-0002-8807-1326, Maracay, Venezuela.

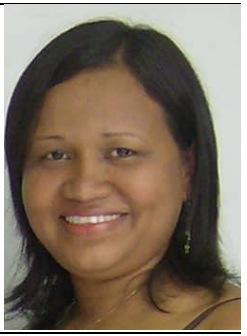




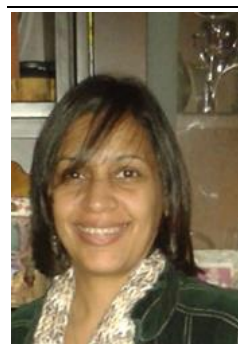

Marling Rosario Brito. Profesora especialista en Dibujo Técnico, con maestría en Educación Mención Procesos del Aprendizaje. Profesora adscrita al Departamento de Ciencia y Tecnología de Comportamiento en la Universidad Simón Bolívar. Profesora en la maestría de Gerencia Educativa de la Universidad Pedagógica Experimental Libertador. Línea de Investigación: estilos de Aprendizaje, Estilos educativos Parentales, Procesos del Aprendizaje, Habilidades Gerenciales Blandas: Optimización del Tiempo y la Toma de Decisiones en la Resolución de problemas. marlingrosario@usb.ve

Martha Iglesias. Universidad Pedagógica Experimental Libertador, Instituto Pedagógico de Maracay, Venezuela. Profesora de Matemática, con Maestría en Enseñanza de la Matemática y Doctorado en Educación. Coordinadora de la Línea de Investigación en Pensamiento Geométrico y Didáctica de la Geometría adscrita al Centro de Investigación en Enseñanza de la Matemática Usando Nuevas Tecnologías. Integrante del Núcleo de Investigación en Educación Matemática.mmiglesias@gmail.com
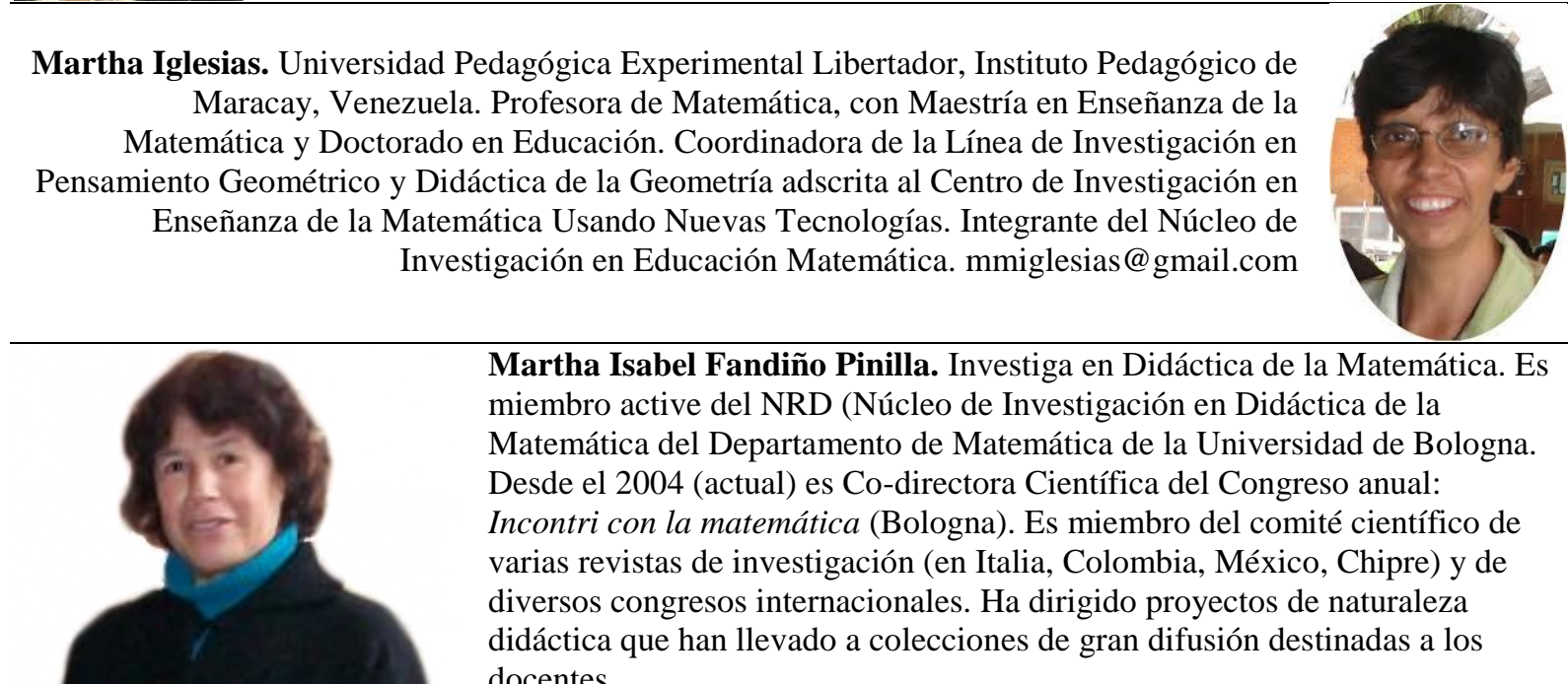

Martha Isabel Fandiño Pinilla. Investiga en Didáctica de la Matemática. Es miembro active del NRD (Núcleo de Investigación en Didáctica de la Matemática del Departamento de Matemática de la Universidad de Bologna. Desde el 2004 (actual) es Co-directora Científica del Congreso anual: Incontri con la matemática (Bologna). Es miembro del comité científico de varias revistas de investigación (en Italia, Colombia, México, Chipre) y de diversos congresos internacionales. Ha dirigido proyectos de naturaleza didáctica que han llevado a colecciones de gran difusión destinadas a los docentes.

Masami Isoda, PhD / Prof, Facultad de Ciencias Humanas / Director, Centro de Investigación sobre Cooperación Internacional en Desarrollo

Educativo, Universidad de Tsukuba, Japón. Ha sido supervisor de coproyectos (representante) del Proyecto de Estudio de Lecciones APEC (desde 2006) y trabaja a nivel internacional y nacional para la innovación de la educación matemática. Estableció las teorías del enfoque de la enseñanza, como la planificación de la argumentación en el aula utilizando la teoría del conocimiento conceptual y de procedimiento en matemáticas.
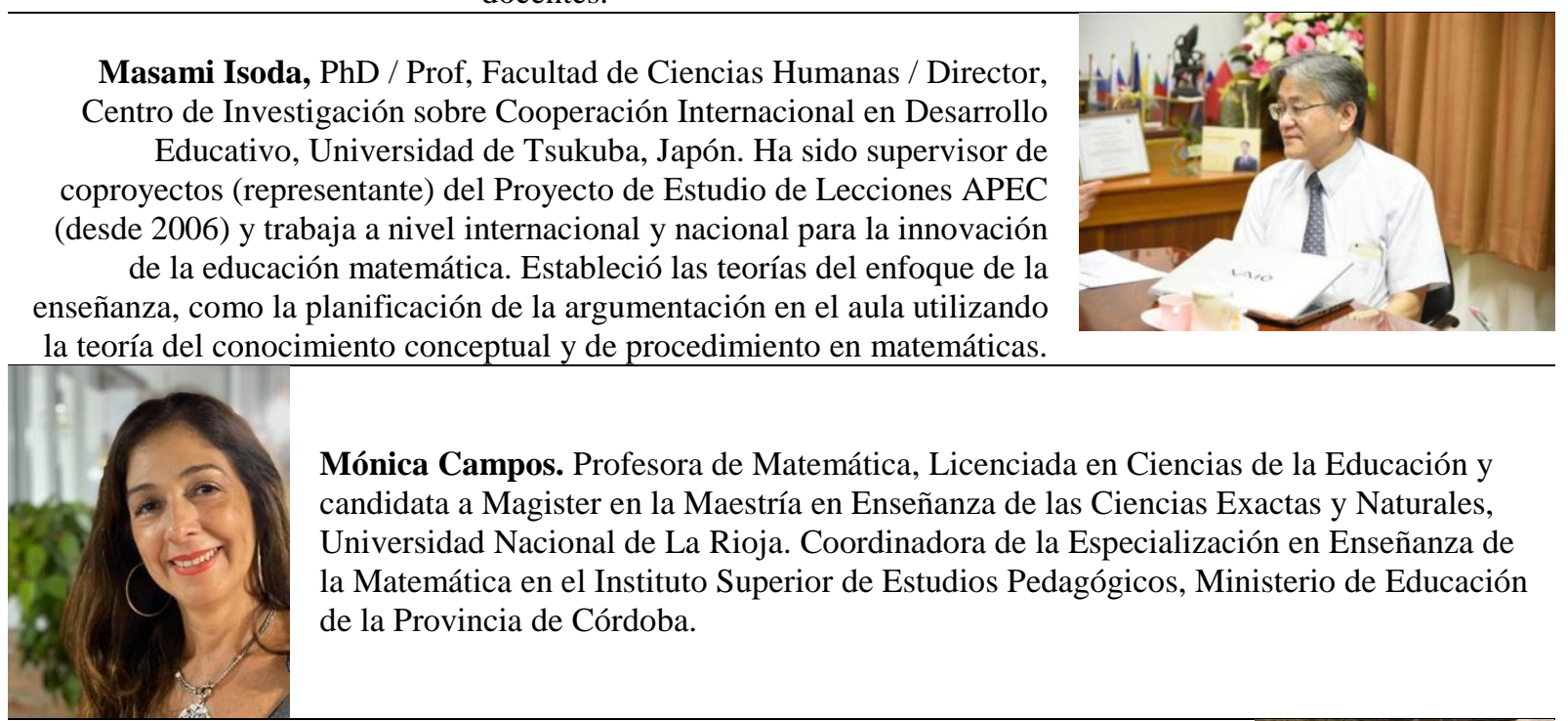

Mónica Campos. Profesora de Matemática, Licenciada en Ciencias de la Educación y candidata a Magister en la Maestría en Enseñanza de las Ciencias Exactas y Naturales, Universidad Nacional de La Rioja. Coordinadora de la Especialización en Enseñanza de la Matemática en el Instituto Superior de Estudios Pedagógicos, Ministerio de Educación de la Provincia de Córdoba.

Nancy Montes de Oca Recio es profesora Titular del Centro de Estudios "Enrique José Varona "de la Universidad de Camagüey "Ignacio Agramonte y Loynaz", Doctora en Ciencias Pedagógicas y Máster en Investigación Educativa. Coordina el grupo de investigación denominado "Perfeccionamiento de las didácticas particulares" de la Universidad de Camagüey y pertenece al grupo científico de Matemática Educativa de la referida universidad. Ha recibido diversos premios entre los que se destacan el Premio de la Academia de Ciencias de la República de Cuba como co-autora en el trabajo "Contribución teórica y práctica del Cálculo Diferencial y del Álgebra Lineal para carreras de Ingeniería" en el año 2019 y como autora principal por la investigación titulada “Aportes teóricos y prácticos a la formación didáctico-matemática de docentes" en el 2020.

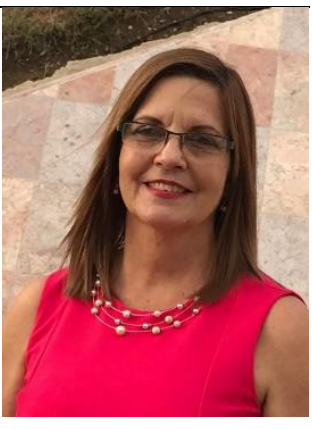




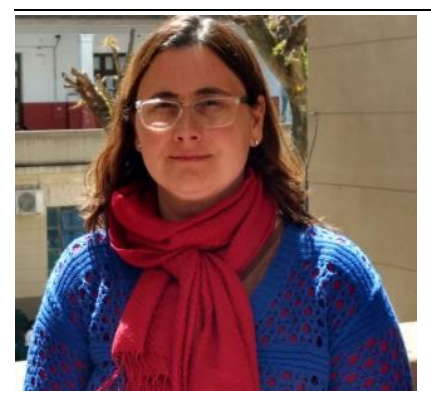

Natalia Sgreccia. Profesora de Enseñanza Media y Superior en Matemática (UNR, 2002). Magíster en Didácticas Específicas con mención en el área Matemática (UNL, 2007). Doctora en Humanidades y Artes con mención en Ciencias de la Educación (UNR, 2012). Tesis: La geometría del espacio en el Profesorado en Matemática: la generación de puentes entre la formación disciplinar y didáctica. Actualmente se encuentra realizando el Programa de Posdoctoración de la Universidad Nacional de Rosario (UNR, 2018-2020). Investigación: El campo de formación en la Práctica Profesional Docente en los Profesorados en Matemática de las Universidades Nacionales

Neide de Almeida Lança Galvão Favaro. Doutorado em Educação (UFSC), Mestrado em Educação (UEM), Professora do Colegiado de Pedagogia da Universidade Estadual do Paraná (UNESPAR), Campus Paranavaí, Coordenadora do Grupo de Estudos e Pesquisas Trabalho e Educação na Sociabilidade do Capital (GEPTESC/UNESPAR/CNPQ) e Pesquisadora do Grupo de Estudos Capital, Trabalho e Educação (GECATE/UFSC/CNPQ).

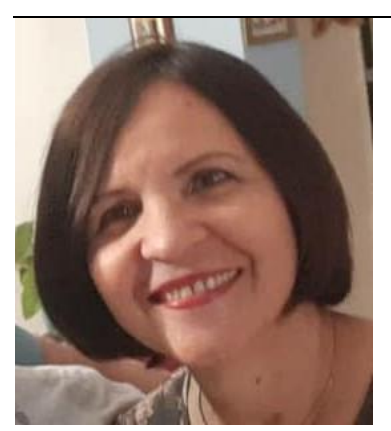

Nelly León Gómez: Profesora de Matemática egresada del Instituto Pedagógico de Maturín, Magister en Estadística Aplicada por la Universidad de Pittsburgh y Magister en Educación por la UPEL-IPM. Presidenta fundadora de la Asociación Venezolana de Educación Matemática (ASOVEMAT). Investigadora adscrita al Núcleo de Investigación de Educación Matemática de la UPEL-IPM (NIEMAT), siguiendo líneas de investigación en Educación Estadística y Formación del docente de Matemática. Sub-directora de la Red de Educación Matemática de América Central y el Caribe (REDUMATE); representante por Venezuela ante el Comité Interamericano de Educación Matemática (CIAEM); miembro del comité editorial de la revista Cuadernos de Investigación y Formación Docente.

Nolberto Goncalves Rodríguez. Doctor en Innovaciones Educativas. Ingeniero de Telecomunicaciones. Profesor Titular en la Facultad de Ciencias de la Educación de la Universidad de Carabobo. Facilitador de Programas de Postgrado. Investigador PEII, Miembro de la Unidad de Investigación en Educación a Distancia. Ponente y Conferencista en eventos académicos. Autor y Árbitro de publicaciones en revistas indizadas. Tutor de Trabajos de Investigación en Pregrado y Postgrado. Diseñador y Mediador de cursos virtuales. Investigador sobre las TIC en las Organizaciones y el Proceso Educativo.

Olga Lidia Pérez González, Doctora en Ciencias Pedagógicas, Profesora Titular del Departamento de Matemática, Investigadora Titular del Centro de Estudios de Ciencias de la Educación "Enrique José Varona", Jefa del Grupo de investigaciones de

Matemática Educativa, Universidad de Camagüey Ignacio Agramonte Loynaz, Premio Nacional de la Academia de Ciencias de Cuba y de la Sociedad Cubana de Matemática y Computación, Presidenta del Comité Latinoamericano de Matemática Educativa (20162020), olguitapg@gmail.com, https://orcid.org/0000-0003-4475-814X, Camagüey, Cuba
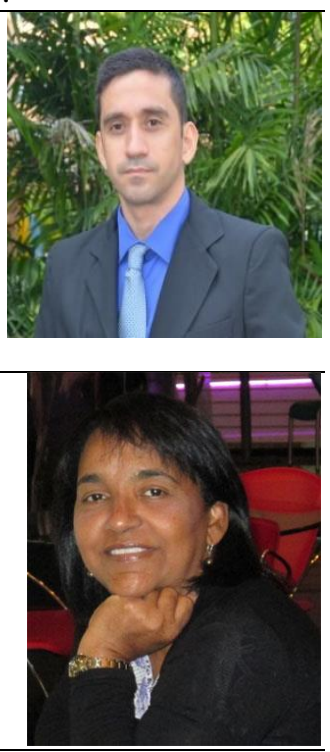
Osvaldo Jesús Rojas Vásquez. Doctor en Ciencias Pedagógicas. Coordinador de los programas de Doctorado y Maestría en Educación Matemática de la Universidad

Antonio Nariño, Colombia. Secretario científico del Simposio de Matemática y

Educación Matemática. Universidad Antonio Nariño. Carrera 38 No. 58A-77, Nicolas de Federman; Bogotá, Colombia.

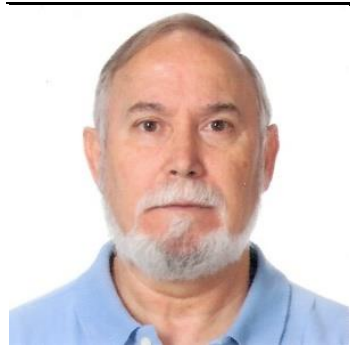

Pablo Arnáez Muga. Profesor Titular Jubilado de la UPEL (Venezuela Maracay). Investigador y docente en el área de la Lingüística en los Postgrados de la UPEL y de la UC (Valencia). Jefe del Departamento de Lengua y Literatura; Coordinador de la Maestría en Lingüística y del Centro de Investigaciones Lingüísticas y Literarias. Ganador de: IV Concurso Experiencias Creativas en Educación; Premio a la Labor Investigativa y Premio CONABA. Miembro del Programa de Promoción del Investigador y de la Cátedra UNESCO (Venezuela). Conferencista y ponente en eventos nacionales e internacionales.pam3346@ hotmail.com

Priscila Augusta de Quadros Scott Hood. Mestrado em Ensino de Ciências e Matemática pela Universidade Luterana do Brasil -ULBRA. Graduação em Matemática pela Universidade Luterana do Brasil. Doutoranda do Programa de Pós-Graduação em Ensino de Ciências e Matemática - PPGECIM da Universidade Luterana do Brasil. Orcid: https://orcid.org/0000-0002-9496-397X. E-mail: prihood@gmail.com

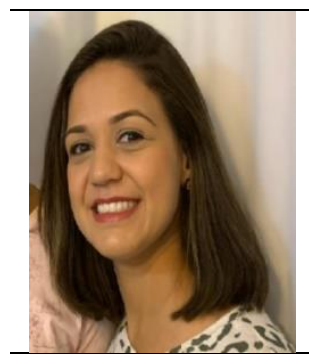

Priscila Semzezem. Doutoranda em Serviço Social (UFSC), Mestrado em Serviço Social e Políticas Sociais (UEL), Professora do Colegiado de Serviço Social da Universidade Estadual do Paraná (UNESPAR), Campus Paranavaí, e Vice-Coordenadora do Grupo de Estudos e Pesquisas Trabalho e Educação na Sociabilidade do Capital (GEPTESC/UNESPAR/CNPQ).

Raimundo Olfos. Es profesor de Matemáticas por la Pontificia Universidad Católica de Valparaíso (PUCV), Chile y Doctor en Filosofía por la Universidad de Gales, Reino Unido. Realizó estudios Post doctorales en la Universidad de Londres, Inglaterra y se especializó en Estudio de Clases para el desarrollo profesional docente en la Universidad de Tsukuba, Japón. El profesor Olfos integra el Claustro de Doctorado en Didáctica de la Matemática que ofrece la PUCV, dirigiendo la línea de Formación de Profesores, además dirige el Grupo de Estudio de Clases de la PUCV, es profesor Asociado del Centro de Investigación Avanzada en Educación, en consorcio entre las universidades PUCV, Universidad de Concepción y Universidad de Chile, y actualmente es presidente de la Sociedad Chilena de Educación Matemática.
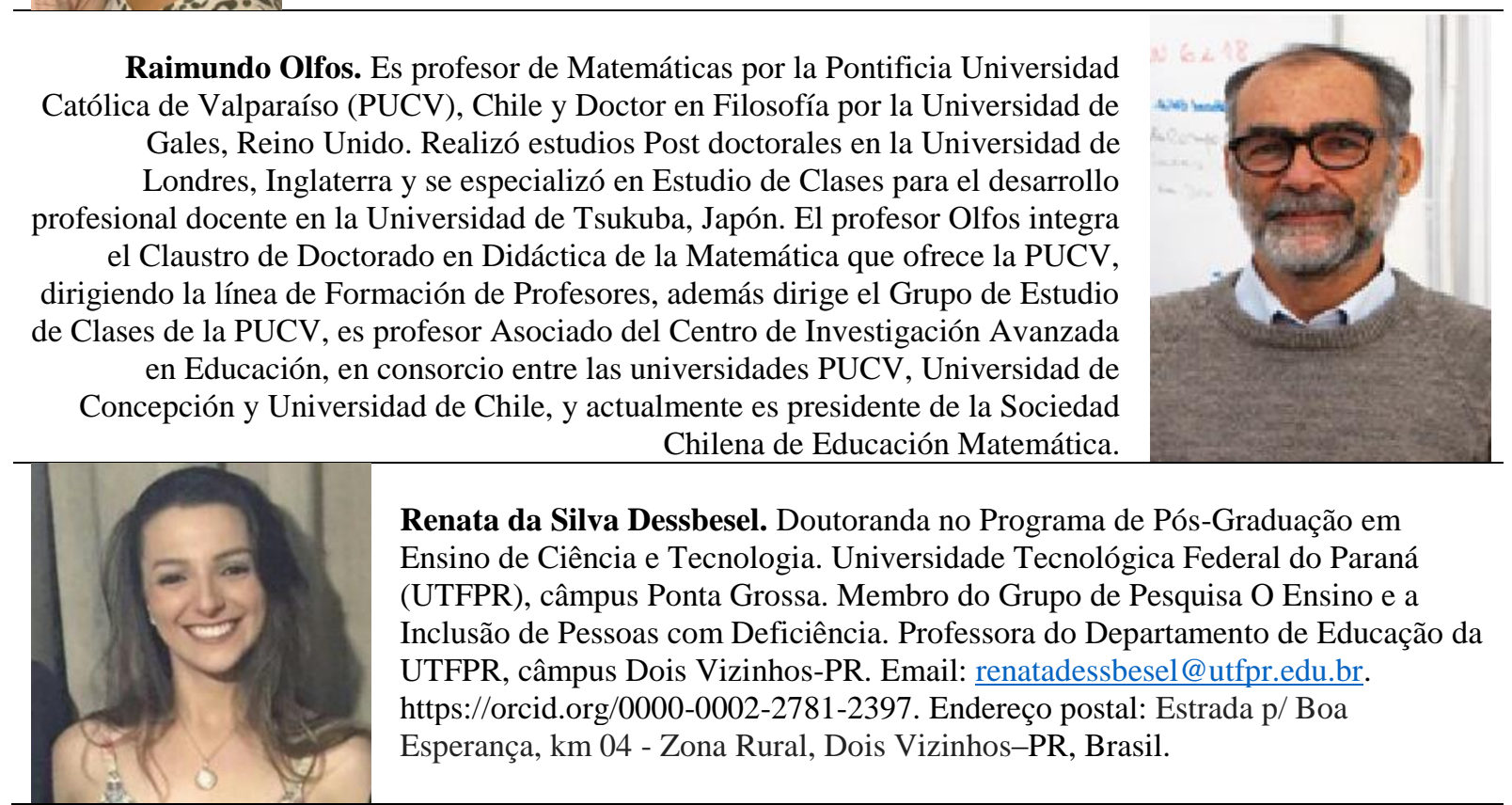

Renata da Silva Dessbesel. Doutoranda no Programa de Pós-Graduação em Ensino de Ciência e Tecnologia. Universidade Tecnológica Federal do Paraná (UTFPR), câmpus Ponta Grossa. Membro do Grupo de Pesquisa O Ensino e a Inclusão de Pessoas com Deficiência. Professora do Departamento de Educação da UTFPR, câmpus Dois Vizinhos-PR. Email: renatadessbesel@utfpr.edu.br. https://orcid.org/0000-0002-2781-2397. Endereço postal: Estrada p/ Boa Esperança, km 04 - Zona Rural, Dois Vizinhos-PR, Brasil. 
Rocío Álvarez Arroyo. Profesora Ayudante Doctora en el Departamento de Didáctica de la Matemática de la Universidad de Granada. Doctora en Ingeniería Civil, Máster en Didáctica de la Matemática, Máster en investigación y avances en microbiología, Ingeniera Química e Ingeniera Técnico Industrial. Cuenta con diversos trabajos publicados en revistas de impacto en el campo de la educación matemática y estadística, ha presentado numerosas comunicaciones en congresos nacionales e internacionales y participa en proyectos de investigación del área.
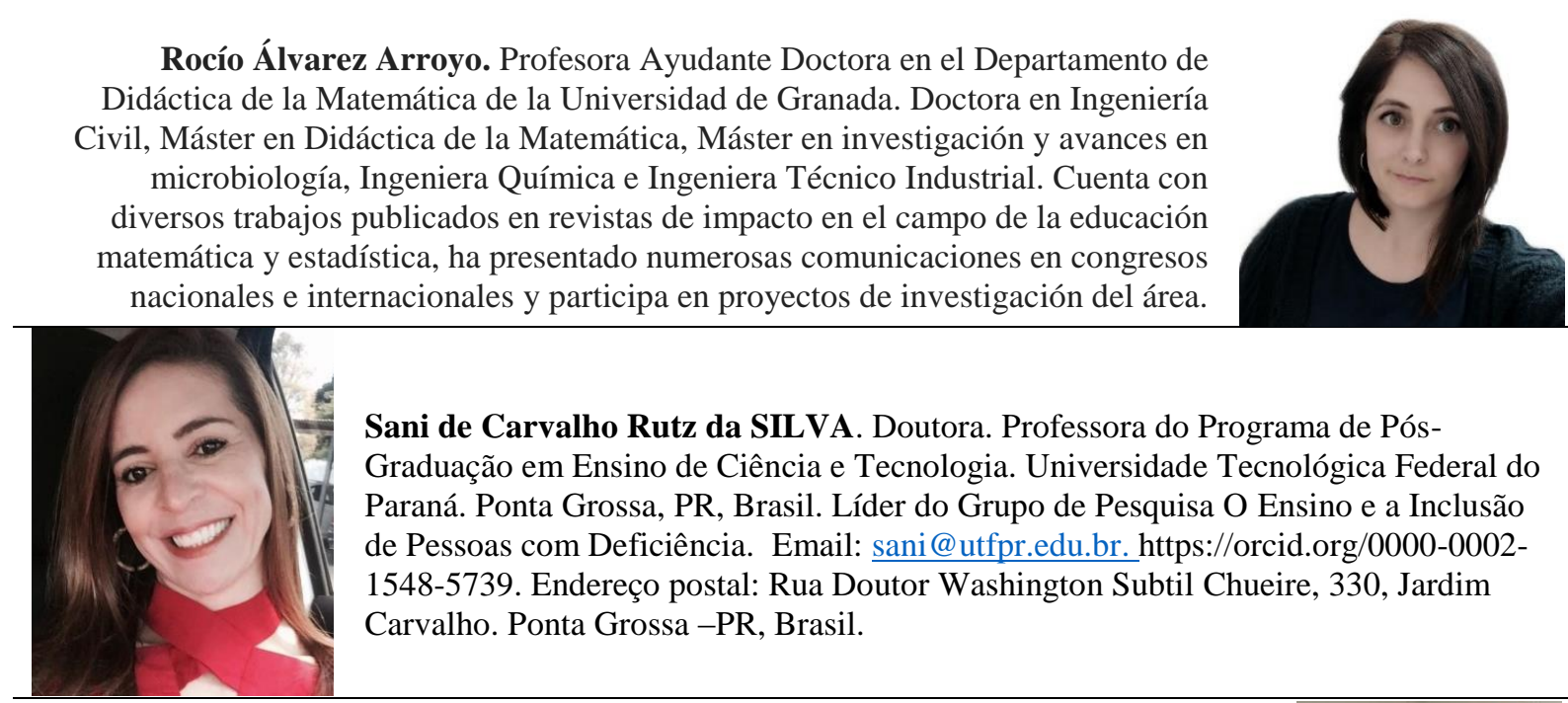

Sani de Carvalho Rutz da SILVA. Doutora. Professora do Programa de PósGraduação em Ensino de Ciência e Tecnologia. Universidade Tecnológica Federal do Paraná. Ponta Grossa, PR, Brasil. Líder do Grupo de Pesquisa O Ensino e a Inclusão de Pessoas com Deficiência. Email: sani@utfpr.edu.br. https://orcid.org/0000-00021548-5739. Endereço postal: Rua Doutor Washington Subtil Chueire, 330, Jardim Carvalho. Ponta Grossa-PR, Brasil.

Silvia Retamal Cisterna, doctora en Educación por la Universidad Autónoma de Barcelona, España. Magister en Educación por la Universidad de Concepción, Chile. Actualmente Académica del Departamento de Educación de la Universidad de Los Lagos, Chile. Desarrolla investigación en el área de la Pedagogía Crítica, Educación Rural y Educación Intercultural.

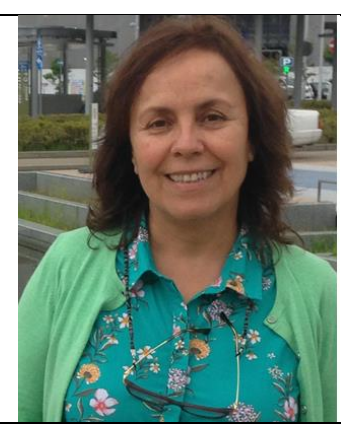

Soledad Estrella. Se doctoró como didacta de la matemática, Su investigación se centra en la Estadística Temprana y el desarrollo del Sentido del Dato. Paralelamente, investiga en la enseñanza y el desarrollo profesional de los profesores de matemáticas a través de Lesson Study, promoviendo la estadística y la educación STEAM mediante clases públicas a nivel regional; es cofundadora del Grupo de Estudio de Clases del IMA. Actualmente conforma el Directorio de la Sociedad Chilena de Estadística; y es representante de Chile ante la CIAEM y miembro activo del ISLP.

Sonia Salas Salinas. Titular de la Unidad Técnico Pedagógica en la Corporación Municipal de Quilpué. Coordinadora comunal de la Red de Didáctica de las Matemáticas. Doctora en Educación por la Universidad de Granada. Más de 20 años trabajando en la formación matemáticas de niños, niñas y adolescentes, en escuelas municipales, con altos índices de vulnerabilidad social en Chile. Trabaja en la formación continua de los docentes en ejercicio y apoyo a los equipos directivos, en didáctica de las matemáticas, para la Corporación Municipal del Quilpué. sbsalass.doc@gmail.com https://orcid.org/0000-0001-6888-7638

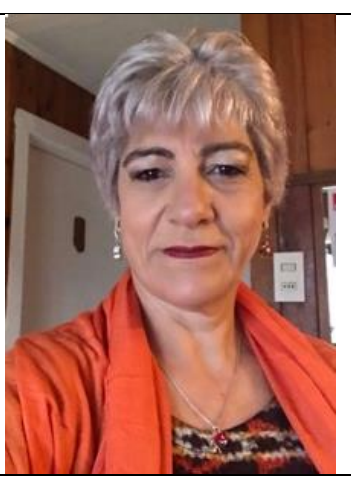




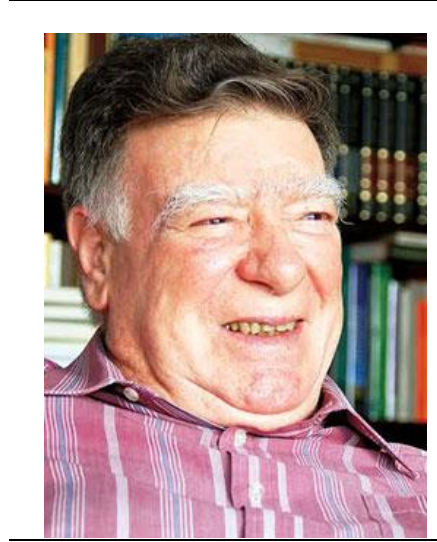

Ubiratan D’Ambrosio. Possui graduação em Matemática pela Universidade de São Paulo (1955) e doutorado em Matemática pela Universidade de São Paulo (1963). É Professor Emérito da Universidade Estadual de Campinas/UNICAMP. Atualmente é Docente Permanente do Programa de PósGraduação em Educação Matemática da Universidade Anhanguera de São Paulo/UNIAN. É também Professor Credenciado dos Programas de PósGraduação em História da Ciência da Pontifícia Universidade Católica de São Paulo, em Educação Faculdade de Educação/FE da Universidade de São Paulo/USP e em Educação Matemática do Instituto de Geociências e Ciências Exatas/IGCE da Universidade Estadual Paulista Júlio de Mesquita Filho/UNESP-Rio Claro. Tem atuado principalmente nos temas: História e Filosofia da Matemática, História e Filosofia das Ciências, Etnomatemática, Etnociência, Educação Matemática e Estudos Transdisciplinares.

Verónica Díaz Quezada. Doctora en Educación con Especialización en Matemáticas. Máster en Evaluación Educacional y Profesora de Matemáticas. Académica Titular e investigadora del Departamento de Ciencias Exactas de la Universidad de Los Lagos, Osorno, Chile. Líneas de investigación: Resolución de problemas; Evaluación en matemáticas; Competencias matemáticas y profesionales. Participación en proyectos nacionales de CONICYT, internacional de la Cátedra Andrés Bello. Consultora matemática para la UNESCO y evaluadora de pregrado y posgrado para la Comisión Nacional de Acreditación CNA de las universidades de Chile. Fundadora del Doctorado en Educación Matemática y del Magister en Educación Matemática en la Universidad de Los Lagos..
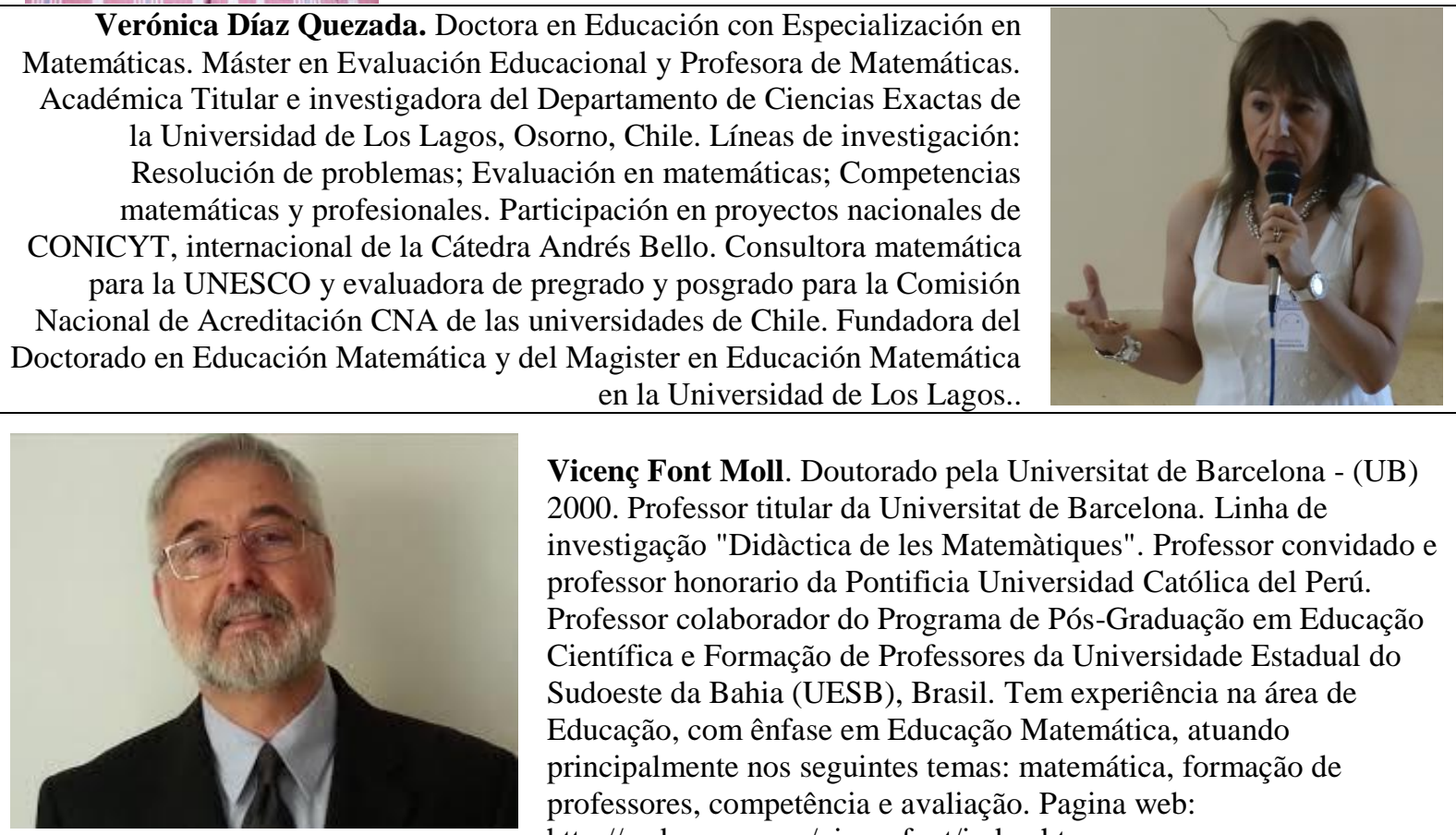

Vicenç Font Moll. Doutorado pela Universitat de Barcelona - (UB) 2000. Professor titular da Universitat de Barcelona. Linha de investigação "Didàctica de les Matemàtiques". Professor convidado e professor honorario da Pontificia Universidad Católica del Perú. Professor colaborador do Programa de Pós-Graduação em Educação Científica e Formação de Professores da Universidade Estadual do Sudoeste da Bahia (UESB), Brasil. Tem experiência na área de Educação, com ênfase em Educação Matemática, atuando principalmente nos seguintes temas: matemática, formação de professores, competência e avaliação. Pagina web: $\mathrm{http}: / /$ webs.ono.com/vicencfont/index.htm

Wagner Valente. É doutor em Educação pela Universidade de São Paulo/ INRP- Paris (1997). Pós-Doutor pela Pontifícia Universidade Católica de São Paulo (1999). Livre Docente no Departamento de Educação da Universidade Federal de São Paulo (2010). Coordenador do GHEMAT - Grupo de Pesquisa de História da Educação Matemática (www.unifesp.br/centros/ghemat). Editor da HISTEMAT - Revista de História da Educação Matemática. Editor do Boletim Acervo, do Centro de Documentação do GHEMAT-SP. Presidente do GHEMAT Brasil.

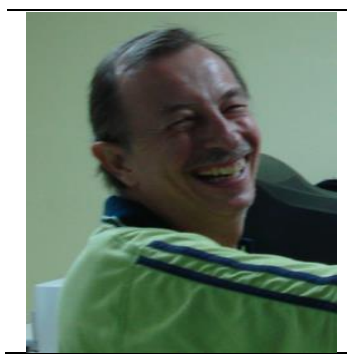

Walter O. Beyer K. Doctor en Educación con énfasis en Educación Matemática y matemático egresado de la Universidad Central de Venezuela. Profesor Asociado jubilado de la Universidad Nacional Abierta. Miembro fundador e integrante del Grupo de Investigación y Difusión en Educación Matemática (GIDEM). Mi principal línea de investigación es la de la Historia de la Matemática y de la Educación Matemática en Venezuela. Correo-e: nowarawb@gmail.com. ORCID Id: 0000-00031726-7994. Caracas, Venezuela. 
Wanda C. Rodríguez Arocho. Posee un doctorado en Filosofía con especialidad en Psicología y una Maestría en Educación con especialidad en Orientación y Consejería, ambos grados otorgados por la Universidad de Puerto Rico. Es Catedrática jubilada del Departamento de Psicología de dicha institución. Su línea de investigación se ha enfocado en procesos cognitivos, aprendizaje, enseñanza de la psicología y desarrollo humano desde la perspectiva histórico-cultural.

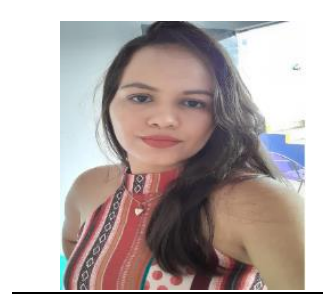

Weslaine da Silva Santos. Graduação em Licenciatura em Biologia pelo Instituto Federal de Mato Grosso (IFMT). Técnica em Alimentos pelo IFMT Campus Confresa. Articuladora de Núcleo local na ONG ENGAJAMUNDO. Representante da sociedade civil no Conselho municipal de meio ambiente de Confresa (CONSEMMA). Atualmente é auxiliar legislativo - Câmara Municipal de Confresa.https://orcid.org/0000-0003-0347$\underline{7122}$

Yohana Taise Hoffmann. Doutoranda pelo Programa de Pós graduação em Educação Científica e Tecnológica (PPGECT) da Universidade Federal de Santa Catarina (UFSC). Mestra em Educação Científica e Tecnológica pela UFSC com estudos no área da História da Educação Matemática. Bacharel e Licenciada em Ciências Sociais pela UFSC, na área da Sociologia da Educação. É integrante do Laboratório de Pesquisas Sociológicas Pierre Bourdieu (LAPSB), do Grupo de Pesquisa Ensino e Formação de Educadores em Santa Catarina (GPEFESC), do Grupo de Pesquisa de História da Educação Matemática - Santa Catarina (GHEMAT-SC) e do Grupo de História da Educação Matemática (GHEMAT - 